\title{
Higher poles and crossing phenomena from twisted genera
}

\author{
Sujay K. Ashok, ${ }^{a}$ Eleonora Dell'Aquila ${ }^{a}$ and Jan Troost $^{b}$ \\ ${ }^{a}$ Institute of Mathematical Sciences, \\ C.I.T Campus, Taramani, Chennai, 600113 India \\ ${ }^{b}$ Laboratoire de Physique Théorique, ${ }^{1}$ Ecole Normale Supérieure, \\ 24 rue Lhomond, F-75231 Paris Cedex 05, France \\ E-mail: sashok@imsc.res.in, edellaquila@imsc.res.in, troost@lpt.ens.fr
}

ABSTRACT: We demonstrate that Appell-Lerch sums with higher order poles as well as their modular covariant completions arise as partition functions in the cigar conformal field theory with worldsheet supersymmetry. The modular covariant derivatives of the elliptic genus of the cigar give rise to operator insertions corresponding to (powers of) right-moving momentum, left-moving fermion number, as well as a term corresponding to an ordinary zero mode partition sum. To show this, we demonstrate how the right-moving supersymmetric quantum mechanics (and in particular the Hamiltonian and spectral density) depend on the imaginary part of the chemical potential for angular momentum. A consequence of our analysis is that varying the imaginary part of the chemical potential for angular momentum on the cigar gives rise to a wall-crossing phenomenon in the bound state contribution to the elliptic genus, while the full elliptic genus is a continuous function of the chemical potential.

Keywords: Conformal Field Models in String Theory, Extended Supersymmetry, Conformal and W Symmetry

ARXIV EPRINT: 1404.7396

\footnotetext{
${ }^{1}$ Unité Mixte du CNRS et de l'Ecole Normale Supérieure associée à l'université Pierre et Marie Curie 6, UMR, 8549
} 


\section{Contents}

1 Introduction 1

2 Path integral elliptic genera 3

2.1 The path integral expression 3

2.2 Modularity and periodicity 4

2.3 The Liouville elliptic genus 5

2.4 The Hamiltonian perspective 5

$\begin{array}{lll}2.5 & \text { The relation to completed Appell-Lerch sums } & 7\end{array}$

2.5.1 Review of the completed Appell-Lerch sum $\widehat{A} \quad 7$

$\begin{array}{ll}2.6 & \text { Supersymmetric quantum mechanics and wall crossing } \\ \end{array}$

3 A model for higher order Appell-Lerch sums $\quad 12$

$\begin{array}{lll}3.1 & \text { Modular covariant derivatives } & 12\end{array}$

$\begin{array}{lll}3.2 & \text { Completed Appell-Lerch sums as state space sums } & 13\end{array}$

$\begin{array}{ll}3.3 & \text { Higher order Appell-Lerch sums and their completion } \\ 3.4\end{array}$

$\begin{array}{ll}3.4 & \text { Generalized elliptic genera } \\ \end{array}$

4 Conclusions $\quad 18$

$\begin{array}{ll}\text { A The path integral of the axially gauged coset model } & 20\end{array}$

$\begin{array}{ll}\text { B Towards the Hamiltonian viewpoint } & 21\end{array}$

$\begin{array}{ll}\text { C Supersymmetric quantum mechanics } & 24\end{array}$

$\begin{array}{ll}\text { D Useful formulae } & 26\end{array}$

\section{Introduction}

Two-dimensional conformal field theories are of physical as well as mathematical interest. One of many ways in which these physical theories connect to mathematics is through the calculation of their elliptic genera. In the case of compact target space manifolds for twodimensional non-linear sigma-models, the elliptic genera capture a plethora of Dirac indices on symmetrized and anti-symmetrized tangent vector bundles of the target space [1].

Elliptic genera can be defined for two-dimensional conformal field theories which have at least one right-moving supercharge. For the right-movers, one mimics the definition of the Witten index, while computing the partition sum for left-movers, twisted by all charges which commute with the right-moving supercharge. 
In this paper, we discuss further aspects of the elliptic genus in a two-dimensional supersymmetric cigar conformal field theory, with an $N=(2,2)$ superconformal symmetry algebra. The elliptic genus, twisted by a global angular momentum charge $P$, is defined as the following trace over the Ramond-Ramond sector Hilbert space $\mathcal{H}$ :

$$
\chi(\tau, \alpha, \beta)=\operatorname{Tr}_{\mathcal{H}}(-1)^{F_{L}+F_{R}} q^{L_{0}-\frac{c}{24}} z^{J_{0}^{R}} y^{P},
$$

where we used the notation $q=e^{2 \pi i \tau}, z=e^{2 \pi i \alpha}$ and $y=e^{2 \pi i \beta}$. The operator $J_{0}^{R}$ measures the left moving $\mathrm{U}(1) R$ charge while $P$ measures the global angular momentum of the states. ${ }^{1}$

The elliptic genera of the supersymmetric Liouville and cigar theories were calculated in [2-4] using the path integral formulation of these theories (see also [5-7]). In particular, we will look at the three variable Jacobi form analyzed in [4] and generalize this result by deriving a path integral expression for the elliptic genus with complex chemical potentials for the R-charge and the global charge. This generalization is natural from a mathematical point of view given that elliptic genera are Jacobi forms, and their arguments are transformed within the set of complex numbers under elliptic and modular transformations.

Once the chemical potentials are complexified, it becomes straightforward to take modular covariant derivatives. We will show that this allows us to find physical models for certain Appell-Lerch sums with higher order poles [8], within the cigar or Liouville conformal field theory. The covariant derivatives correspond to operator insertions of rightmoving momentum and left-moving fermion number, as well as a contribution from a zero mode partition sum. The right-moving momentum is not strictly conserved, but is a good asymptotic quantum number that can be used to label states in partition sums. We will show that a modification of the supersymmetric quantum mechanics for right-moving primaries is induced by the insertions, which changes the difference in spectral densities arising in the integral over the continuum.

As a result of our analysis, we will encounter a wall-crossing phenomenon in a simple two-dimensional superconformal field theory. By varying the imaginary part of the angular momentum chemical potential $\beta$, some states are subtracted from the discrete part of the spectrum, as coded in the holomorphic part of the elliptic genus, while other states are added to the bound state spectrum. The continuum contribution exhibits a complementary behaviour such that the full elliptic genus is continuous.

Another motivation for our analysis comes from the widening range of applicability of mock modular forms in physics. Most of the early applications focused on restoring a duality or modular invariance $[9,10]$ in identifiable holomorphic contributions to e.g. superconformal characters [11-13] or supergravity partition functions affected by wallcrossing [14]. The modular completion was shown to arise naturally in the context of the superconformal coset partition function calculation [2], which allowed for many generalizations in the two-dimensional realm, and applications to the physics of two-dimensional and higher-dimensional black holes in string theory $[15,16]$. Space-time indices inherit mock modularity of the worldsheet indices [17]. Still, most of the mock modularity of space-time

\footnotetext{
${ }^{1}$ Following thermodynamics nomenclature, we will often refer to $\alpha$ and $\beta$ as chemical potentials.
} 
indices (see e.g $[8,18]$ ) remains poorly understood, and providing microscopic models for mock modular forms in two dimensions (e.g. on the worldsheet of an (effective) string) may well be instrumental in identifying the relevant physics in space-time.

Our paper is structured as follows. We start in section 2 by proposing the path integral expression for the elliptic genus with chemical potentials taking values in the complex plane. We then perform the traditional transform to the Hamiltonian form in order to recover known results in the mathematics literature, and interpret the result in terms of a modified right-moving supersymmetric quantum mechanics. We then exploit the result to exhibit a wall crossing phenomenon as a function of the imaginary part of the chemical potential for angular momentum. ${ }^{2}$ In section 3 we further apply the result to consider modular covariant derivatives of these results. These will serve to model higher order Appell-Lerch sums and to give a direct conformal field theory interpretation of their modular completions, in particular in a Hamiltonian form. We conclude in section 4 with a discussion and suggestions for generalizations and applications.

\section{Path integral elliptic genera}

Elliptic genera, defined as twisted partition functions on tori, have natural elliptic and modular properties; they are Jacobi forms [19]. The modular transformation properties of the three variable elliptic genus of the $N=2$ Liouville conformal field theory we will study are:

$$
\begin{aligned}
\chi(\tau+1, \alpha, \beta) & =\chi(\tau, \alpha, \beta) \\
\chi\left(-\frac{1}{\tau}, \frac{\alpha}{\tau}, \frac{\beta}{\tau}\right) & =e^{\frac{c}{3} \frac{\pi i \alpha^{2}}{\tau}-\frac{2 \pi i \alpha \beta}{\tau}} \chi(\tau, \alpha, \beta) .
\end{aligned}
$$

where $c=3+6 / k$ is the central charge of the theory and we will take the level $k$ to be a positive integer. The genus moreover has periodicity properties in $\alpha$ under shifts by integer multiples of $k(1, \tau)$ and in $\beta$ under shifts by multiples of 1 and $\tau$. Since these elliptic transformations and the modular transformations in (2.1) shift and rescale chemical potentials by the complex parameter $\tau$, it is natural to study elliptic genera for complex chemical potentials. This demands a slight generalization of the analysis in [2-4]. Further physical motivations for this generalization will become clear in the course of the paper.

\subsection{The path integral expression}

In an earlier work [4], we obtained the following expression for the elliptic genus of the cigar superconformal field theory: ${ }^{3}$

$$
\chi_{\cos }(\tau, \alpha, \beta)=\int_{0}^{1} d s_{1,2} \frac{\theta_{11}\left(\tau, s_{1} \tau+s_{2}-\frac{k+1}{k} \alpha+\beta\right)}{\theta_{11}\left(\tau, s_{1} \tau+s_{2}-\frac{\alpha}{k}+\beta\right)} \sum_{m, w \in \mathbb{Z}} e^{-2 \pi i s_{2} w+2 \pi i s_{1}(m-\alpha)} e^{-\frac{\pi}{k \tau_{2}}|m-\alpha+w \tau|^{2}} .
$$

\footnotetext{
${ }^{2}$ We will use the word wall crossing to refer to a jump in the bound state spectrum arising when a modulus crosses a particular value.

${ }^{3}$ See appendix D for the definition and properties of theta-functions.
} 
Modularity and ellipticity of the elliptic genus were exhibited in [4] using this expression. This is therefore the expression that generalizes naturally to the case of complex chemical potentials $\alpha=\alpha_{1}+\tau \alpha_{2}$ and $\beta=\beta_{1}+\tau \beta_{2}$. A double Poisson resummation (and combining holonomy variables and winding numbers) produces the path integral form of the elliptic genus:

$$
\chi_{\cos }(\tau, \alpha, \beta)=k \int_{-\infty}^{+\infty} d s_{1} d s_{2} \frac{\theta_{11}\left(\tau, s_{1} \tau+s_{2}-\frac{k+1}{k} \alpha+\beta\right)}{\theta_{11}\left(\tau, s_{1} \tau+s_{2}-\frac{\alpha}{k}+\beta\right)} e^{-\frac{k \pi}{\tau_{2}}\left|s_{1} \tau+s_{2}\right|^{2}} e^{-2 \pi i \alpha_{2}\left(s_{1} \tau+s_{2}\right)}
$$

Compared with [4], here we have an additional factor whose exponent is proportional to $\alpha_{2}$, since we have now allowed for complex $\alpha$. A path integral derivation of the formula is given in appendix A. It is interesting to observe that this extra factor can be combined in the following way by shifting the integration variable by $\beta-\frac{\alpha}{k}$ :

$$
\chi_{\cos }(\tau, \alpha, \beta)=k \int_{-\infty}^{+\infty} d s_{1} d s_{2} \frac{\theta_{11}\left(\tau, s_{1} \tau+s_{2}-\alpha\right)}{\theta_{11}\left(\tau, s_{1} \tau+s_{2}\right)} e^{-\frac{k \pi}{\tau_{2}}\left(s_{1} \tau+s_{2}+\frac{\alpha}{k}-\beta\right)\left(s_{1} \bar{\tau}+s_{2}+\frac{\alpha}{k}-\bar{\beta}\right)}
$$

This manipulation shows explicitly that the elliptic genus is independent of $\bar{\alpha}$ and depends only holomorphically on the $\alpha$ variable. ${ }^{4}$ Interestingly the elliptic genus is not holomorphic in $\beta$, as one sees in the exponent of the last expression. This property will prove crucial in exhibiting a wall crossing phenomenon in the conformal field theory.

An equivalent, equally useful way to write the path integral answer, is in terms of holonomies $s_{1,2}$ in the interval $[0,1]$ and integer winding numbers $m$ and $w$ :

$$
\begin{array}{rl}
\chi_{\cos }(\tau, \alpha, \beta)=k \sum_{n, m} \int_{0}^{1} d s_{1} & d s_{2} \frac{\theta_{11}\left(\tau, s_{1} \tau+s_{2}-\alpha\right)}{\theta_{11}\left(\tau, s_{1} \tau+s_{2}\right)} e^{2 \pi i \alpha n} \\
& \times e^{-\frac{k \pi}{\tau_{2}}\left|\left(n+s_{1}\right) \tau+m+s_{2}+\frac{\alpha}{k}-\beta\right|^{2}} e^{-2 \pi i \alpha_{2}\left(m+s_{2}+\frac{\alpha}{k}+\tau\left(n+s_{1}\right)-\beta\right)} .
\end{array}
$$

We have shifted the $\alpha$ and $\beta$ dependence of the bosons into the exponential factor. This form of the elliptic genus path integral arises naturally from the derivation in terms of a gauged linear sigma model $[20,21]$. Again, we have generalized the result to complexified $\alpha$ and $\beta$.

\subsection{Modularity and periodicity}

Another strong argument for the generalized path integral expression in (2.3) is that it can now be checked directly, using equation (2.3), to be modular and elliptic. Indeed, invariance under the modular $T$ transformation is easily established by shifting $s_{2}$ appropriately. Under the modular $S$-transformation, the variables transform as

$$
\tau \rightarrow-\frac{1}{\tau} \quad \alpha \rightarrow \frac{\alpha}{\tau} \quad \beta \rightarrow \frac{\beta}{\tau} \quad s_{2} \rightarrow s_{1} \quad s_{2} \rightarrow-s_{1} \quad \alpha_{2} \longrightarrow \tau \alpha_{2}-\alpha .
$$

Using these and the appropriate modular properties of the theta function (see appendix D), one can check that the integrand picks up an exponential factor that matches the modular

\footnotetext{
${ }^{4}$ We would like to thank Sameer Murthy for helpful discussions on this point.
} 
transformation in equation (2.1), with the central charge $c=3+6 / k$ of the supersymmetric cigar theory.

The periodicity properties as well can be checked directly on the path integral expression (2.3). Consider for concreteness the shift $\alpha \rightarrow \alpha+k \tau$. The absolute value term in the exponent of (2.3) is unchanged by this transformation; the last ( $\alpha_{2}$ dependent) factor picks up a contribution $e^{-2 \pi i k\left(s_{1} \tau+s_{2}\right)}$. Combining this with the elliptic property of the theta function recorded in equation (D.2), the integrand of the elliptic genus picks up a combined factor:

$$
\frac{(-1)^{k+1} q^{-\frac{(k+1)^{2}}{2}} q^{(k+1) s_{1}} e^{2 \pi i s_{2}(k+1)} z^{-\frac{(k+1)^{2}}{k}} y^{k+1}}{(-1) q^{-\frac{1}{2}} q^{s_{1}} e^{2 \pi i s_{2}} z^{-\frac{1}{k}} y} e^{-2 \pi i k\left(s_{1} \tau+s_{2}\right)}=(-1)^{k} q^{-\frac{k^{2}+2 k}{2}} z^{-(k+2)} y^{k} .
$$

Using the value of the central charge, we can write the elliptic property of the elliptic genus as

$$
\chi_{\cos }(\tau, \alpha+k \tau, \beta)=(-1)^{\frac{c}{3} k} e^{-\frac{\pi i c}{3}\left(k^{2} \tau+2 k \alpha\right)} e^{2 \pi i \beta k} \chi_{\cos }(\tau, \alpha, \beta) .
$$

Along these lines we can also check the ellipticity under shift of $\alpha$ by integer multiples of $k$, and shifts of $\beta$ by integer multiplies of 1 and $\tau$. The resulting properties are those recorded in [4]. Here, we derived these properties directly from the path integral expression which is now valid for complexified chemical potentials $\alpha$ and $\beta$.

\subsection{The Liouville elliptic genus}

The cigar elliptic genus is related to that of $N=2$ Liouville theory by a $\mathbb{Z}_{k}$ orbifold $[3,4]$. Thus, we find for the Liouville elliptic genus:

$$
\begin{aligned}
\chi_{L}(\tau, \alpha, \beta)=\sum_{n, m} \int_{0}^{1} d s_{1} d s_{2} \frac{\theta_{11}\left(\tau, s_{1} \tau+s_{2}-\alpha\right)}{\theta_{11}\left(\tau, s_{1} \tau+s_{2}\right)} e^{2 \pi i \alpha \frac{n}{k}} e^{-\frac{k \pi}{\tau_{2}}\left|\left(\frac{n}{k}+s_{1}\right) \tau+\left(\frac{m}{k}+s_{2}\right)+\frac{\alpha}{k}-\beta\right|^{2}} \\
\times e^{-2 \pi i \alpha_{2}\left(\left(\frac{n}{k}+s_{1}\right) \tau+\left(\frac{m}{k}+s_{2}\right)+\frac{\alpha}{k}-\beta\right)} .
\end{aligned}
$$

The Liouville elliptic genus is most simply related [2] to the single pole Appell-Lerch sum studied in the mathematics literature [9, 12]. Since we wish to compare our results with this literature, we work in Liouville theory in what follows. All our statements apply, mutatis mutandis, to orbifolds of Liouville theory, tensor product theories, et cetera. In particular, they have straightforward equivalents for the two-dimensional black hole superconformal field theory.

\subsection{The Hamiltonian perspective}

It is instructive to understand the sum over states coded in the path integral result. In order to reach this perspective, one needs to switch from a Lagrangian to a Hamiltonian viewpoint. This can be achieved after a number of technical steps that were performed in [2-4]; the generalization of these steps to complexified chemical potentials $\alpha$ and $\beta$ is detailed in appendix B. The final result is a sum of two terms, written out in (B.17) 
and (B.21); the first one is a holomorphic, right-moving ground state contribution to the elliptic genus of the form:

$$
\begin{aligned}
& \chi_{L, h o l}(\tau, \alpha, \beta)=\frac{i \theta_{11}(\tau, \alpha)}{\pi \eta^{3}} \sum_{m, v, w} {\left[\int_{\mathbb{R}}-\int_{\mathbb{R}-\frac{i k}{2}}\right] \frac{d s}{2 i s+v-k \beta_{2}} S_{m+k w-1} } \\
& \times z^{v / k-2 w} y^{k w} q^{-v w+k w^{2}}(q \bar{q})^{\left(i s+\frac{v}{2}-\frac{k \beta_{2}}{2}\right)+\frac{s^{2}}{k}+\frac{\left(v-k \beta_{2}\right)^{2}}{4 k}},
\end{aligned}
$$

where $v=n+k w$ represents the right-moving momentum on the asymptotic circle (in terms of the angular momentum $n$ and winding number $w$ ) and $S_{r}$ is a sum introduced in appendix D. The contour integral picks up poles corresponding to right-moving ground state contributions, which are holomorphic. The new ingredient, compared to the earlier works, is the $\beta_{2}$ dependence. We observe that if $k \beta_{2}$ is not an integer, the contour integrals are unambiguously defined. If $k \beta_{2}$ is an integer, we define our integrals parallel to the real line to be shifted slightly, by adding a small imaginary part $\epsilon$ to the integration contour. In all cases, the contours are taken such that we sum the right-moving momentum $v$ over a range of integers, the highest of which is the integer $\left[k \beta_{2}\right]$ smaller or equal to $k \beta_{2}$, and the lowest of which is that integer minus $k-1$. In total, there are $k$ integer valued right-moving momenta $v$ between the two contours. We obtain the expression:

$\chi_{L, h o l}(\tau, \alpha, \beta)=-\sum_{m, w} \sum_{v=-(k-1)+\left[k \beta_{2}\right]}^{\left[k \beta_{2}\right]}(-1)^{m} q^{(m-1 / 2)^{2} / 2} z^{m-1 / 2} S_{m+k w-1} z^{v / k-2 w} y^{k w} q^{k w^{2}-v w}$.

If we define $m=-\tilde{m}+1$ and use the identity (D.4) we find that the holomorphic part of the elliptic genus is given by:

$$
\begin{aligned}
\chi_{L, h o l}(\tau, \alpha, \beta) & =\frac{i \theta_{11}(\tau,-\alpha)}{\eta^{3}(\tau)} \sum_{w} \frac{z^{-2 w} y^{k w} q^{k w^{2}}}{1-z^{-1} q^{k w}}\left(z^{\frac{1}{k}} q^{-w}\right)^{\left[k \beta_{2}\right]} \sum_{v=0}^{k-1}\left(z^{-\frac{1}{k}} q^{w}\right)^{v} \\
& =\frac{i \theta_{11}(\tau,-\alpha)}{\eta^{3}(\tau)} \sum_{w} \frac{z^{-2 w} y^{k w} q^{k w^{2}}}{1-z^{-\frac{1}{k}} q^{w}}\left(z^{\frac{1}{k}} q^{-w}\right)^{\left[k \beta_{2}\right]} \\
& =z^{\left[k \beta_{2}\right] / k} \frac{i \theta_{11}(\tau,-\alpha)}{\eta^{3}(\tau)} \sum_{w} \frac{\left(z^{-2} y^{k} q^{-\left[k \beta_{2}\right]}\right)^{w} q^{k w^{2}}}{1-z^{-\frac{1}{k}} q^{w}} .
\end{aligned}
$$

This is a sum over extended $N=2$ superconformal algebra characters based on Ramond ground states of $\mathrm{R}$-charge $1 / 2-l / k+\left[k \beta_{2}\right] / k$ where $l$ takes values in the set $l=0,1, \ldots, k-1$. This can be seen from the first line, where we identify $w$ as the spectral flow summation variable.

There is also a non-holomorphic contribution to the elliptic genus, which arises from the difference in spectral densities for fermionic and bosonic right-moving primaries:

$$
\chi_{L, r e m}(\tau, \alpha, \beta)=\frac{i \theta_{11}(\tau,-\alpha)}{\pi \eta^{3}(\tau)} \sum_{v, w} \int_{\mathbb{R}} \frac{d s}{2 i s+v-k \beta_{2}} z^{v / k-2 w} y^{k w} q^{k w^{2}-v w}(q \bar{q})^{\frac{s^{2}}{k}+\frac{\left(v-k \beta_{2}\right)^{2}}{4 k}} .
$$


We note that both the holomorphic piece and the remainder term are invariant under the transformation $\beta \rightarrow \beta+1$, and each picks up a factor of $z$ under $\beta \rightarrow \beta+\tau$. These two terms, in (2.12) and (2.13) sum to the modular completion of the Appell-Lerch sum analyzed in the mathematics literature [9], as we show in the next section.

\subsection{The relation to completed Appell-Lerch sums}

In this subsection, we wish to show how the path integral result for the Liouville elliptic genus for complexified arguments, rewritten in the Hamiltonian form, relates to the mathematics of modularly completed Appell-Lerch sums [9, 12].

\subsubsection{Review of the completed Appell-Lerch sum $\widehat{A}$}

The holomorphic Appell-Lerch sum is defined as ${ }^{5}$

$$
\mathcal{A}_{1, k}(\tau, u, v)=a^{k} \sum_{n \in \mathbb{Z}} \frac{q^{k n(n+1)} b^{n}}{1-a q^{n}} .
$$

In the conventions of [9], the three variables are denoted by $q=e^{2 \pi i \tau}, a=e^{2 \pi i u}$ and $b=e^{2 \pi i v}$. The remainder of this Appell-Lerch sum, which, when added to the holomorphic part (2.14) leads to a Jacobi form, is given by [9]

$$
\mathcal{R}_{1, k}(\tau, u, v)=\frac{i}{4 k} a^{\frac{2 k-1}{2}} \sum_{m(m o d) 2 k} \theta_{11}\left(\frac{v+m}{2 k}+\frac{(2 k-1) \tau}{4 k} ; \frac{\tau}{2 k}\right) R\left(u-\frac{v+m}{2 k}-\frac{\tau(2 k-1)}{4 k} ; \frac{\tau}{2 k}\right)
$$

where the function $R$ is defined as:

$$
R(u ; \tau)=\sum_{\nu \in \mathbb{Z}+\frac{1}{2}}\left(\operatorname{sgn}(\nu)-\operatorname{Erf}\left[\sqrt{2 \pi \tau_{2}}\left(\nu+\frac{\operatorname{Im}(u)}{\tau_{2}}\right)\right]\right)(-1)^{\nu-\frac{1}{2}} a^{-\nu} q^{-\frac{\nu^{2}}{2}} .
$$

The sum

$$
\widehat{\mathcal{A}}_{1, k}(\tau, u, v)=\mathcal{A}_{1, k}(\tau, u, v)+\mathcal{R}_{1, k}(\tau, u, v)
$$

satisfies good modular and elliptic properties [9]:

$$
\begin{array}{ll}
\widehat{\mathcal{A}}_{1, k}(\tau, u+1, v)=\widehat{\mathcal{A}}_{1, k}(\tau, u, v) & \widehat{\mathcal{A}}_{1, k}(\tau, u, v+1)=\widehat{\mathcal{A}}_{1, k}(\tau, u, v) \\
\widehat{\mathcal{A}}_{1, k}(\tau, u+\tau, v)=a^{2 k} b^{-2 k} q^{k} \widehat{\mathcal{A}}_{1, k}(\tau, u, v) & \widehat{\mathcal{A}}_{1, k}(\tau, u, v+\tau)=a^{-1} \widehat{\mathcal{A}}_{1, k}(\tau, u, v) \\
\widehat{\mathcal{A}}_{1, k}(\tau+1, u, v)=\widehat{\mathcal{A}}_{1, k}(\tau, u, v) & \widehat{\mathcal{A}}_{1, k}\left(-\frac{1}{\tau}, \frac{u}{\tau}, \frac{v}{\tau}\right)=\tau e^{\frac{2 \pi i}{\tau}\left(v u-k u^{2}\right)} \widehat{\mathcal{A}}_{1, k}(\tau, u, v) .
\end{array}
$$

The map to the conformal field theory variables used earlier is:

$$
u=-\frac{\alpha}{k} \quad v=-2 \alpha-k \tau+k \beta-\tau\left[k \beta_{2}\right],
$$

Through this map, we can rewrite the Appell-Lerch sum in the form:

$$
\mathcal{A}_{1, k}=z^{-1} \sum_{w \in \mathbb{Z}} \frac{q^{k w^{2}}\left(z^{-2} y^{k} q^{-\left[k \beta_{2}\right]}\right)^{w}}{1-z^{-\frac{1}{k}} q^{w}}
$$

\footnotetext{
${ }^{5}$ In [9] the Appell-Lerch sum is denoted $A_{2 k}$. Since we go on to define a series of higher pole Appell-Lerch sums, we use a notation close (but not identical) to [8].
} 
with the usual notation $q=e^{2 \pi i \tau}, z=e^{2 \pi i \alpha}$ and $y=e^{2 \pi i \beta}$. Note that the choice of map (2.19) gives as an immediate match between the holomorphic part of the Appell-Lerch sum and the holomorphic part of the elliptic genus. Matching the remainder terms is less straightforward and it will be the object of the rest of this section.

Applying the map (2.19) to the remainder yields

$$
\mathcal{R}_{1, k}=\frac{i}{4 k} z^{-1+\frac{1}{2 k}} \sum_{m(\bmod ) 2 k} \theta_{11}\left(-\frac{\alpha}{k}+\frac{\beta}{2}-\frac{\tau\left[k \beta_{2}\right]}{2 k}+\frac{m}{2 k}-\frac{\tau}{4 k} ; \frac{\tau}{2 k}\right) R\left(\frac{\tau}{4 k}-\frac{\beta}{2}+\frac{\tau\left[k \beta_{2}\right]}{2 k}-\frac{m}{2 k} ; \frac{\tau}{2 k}\right) .
$$

With these arguments, the function $R$ in (2.16) evaluates to

$$
\begin{aligned}
R=\sum_{\nu \in \mathbb{Z}+\frac{1}{2}}(-1)^{\nu-\frac{1}{2}} y^{\frac{\nu}{2}} q^{-\nu\left[k \beta_{2}\right] / 2 k} q^{-\frac{\nu^{2}}{4 k}-\frac{\nu}{4 k}} e^{\frac{2 \pi i \nu m}{2 k}} & \left(\operatorname{sgn}(\nu)-\operatorname{Erf}\left[\sqrt{\frac{\pi \tau_{2}}{k}}\left(\nu+\frac{1}{2}-\frac{\operatorname{Im}\left(k \beta-\tau\left[k \beta_{2}\right]\right)}{\tau_{2}}\right)\right]\right) \\
=-y^{-\frac{1}{4}} q^{\frac{1}{16 k}} & \sum_{r \in \mathbb{Z}}(-1)^{r} y^{\frac{r}{2}} q^{-r\left[k \beta_{2}\right] / 2 k} q^{\left[k \beta_{2}\right] / 4 k} q^{-\frac{r^{2}}{4 k}} e^{\frac{2 \pi i\left(r-\frac{1}{2}\right) m}{2 k}} \\
& \times\left(\operatorname{sgn}\left(r-\frac{1}{2}\right)-\operatorname{Erf}\left[\sqrt{\frac{\pi \tau_{2}}{k}}\left(r-\frac{\operatorname{Im}\left(k \beta-\tau\left[k \beta_{2}\right]\right)}{\tau_{2}}\right)\right]\right) .
\end{aligned}
$$

The $\theta_{11}$ function that appears in (2.21) evaluates to

$\theta_{11}\left(-\frac{\alpha}{k}+\frac{\beta}{2}-\frac{\tau\left[k \beta_{2}\right]}{2 k}+\frac{m}{2 k}-\frac{\tau}{4 k} ; \frac{\tau}{2 k}\right)=i \sum_{n \in \mathbb{Z}}(-1)^{n} q^{\frac{n^{2}}{4 k}} q^{-\frac{1}{16 k}} z^{\frac{n}{k}-\frac{1}{2 k}} y^{-\frac{n}{2}+\frac{1}{4}} q^{\frac{\tau\left[k \beta_{2}\right]}{2 k}\left(n-\frac{1}{2}\right)} e^{\frac{2 \pi i m}{2 k}\left(\frac{1}{2}-n\right)}$.

Multiplying the two expressions in equations (2.22) and (2.23), all the constant exponents of $q, y$ and $z$ cancel except for one factor of $z$. Furthermore, the variable $m$ can be summed to give

$$
\sum_{m(m o d) 2 k} e^{\frac{2 \pi i m}{2 k}(r-n)}=2 k \delta_{n-r+2 k \mathbb{Z}} .
$$

Substituting the solution to the constraint equation $n=r+2 k j$, where $j \in \mathbb{Z}$, one notices that the summation over $j$ results in a theta function with indices $(r, k)$ (see equation (D.5)). Gathering all these results leads to a more compact expression for the remainder term:

$$
\begin{array}{r}
\mathcal{R}_{1, k}=\frac{z^{-1}}{2} \sum_{r \in \mathbb{Z}}\left(\operatorname{sgn}\left(r-\frac{1}{2}\right)-\operatorname{Erf}\left[\sqrt{\frac{\pi \tau_{2}}{k}}\left(r-\frac{\operatorname{Im}\left(k \beta-\tau\left[k \beta_{2}\right]\right)}{\tau_{2}}\right)\right]\right) y^{\frac{r}{2}} q^{-\frac{r\left[k \beta_{2}\right]}{2 k}} q^{-\frac{r^{2}}{4 k}} \\
\times \theta_{r, k}\left(\tau, \frac{\alpha}{k}-\frac{\beta}{2}+\frac{\tau\left[k \beta_{2}\right]}{2 k}\right) .
\end{array}
$$

Flipping the sign of $r$ and using the fact that both the sign and error functions are odd, we obtain:

$$
\begin{aligned}
& \mathcal{R}_{1, k}=-\frac{z^{-1}}{2} \sum_{r \in \mathbb{Z}}\left(\operatorname{sgn}\left(r+\frac{1}{2}\right)-\operatorname{Erf}\right. {\left.\left[\sqrt{\frac{\pi \tau_{2}}{k}}\left(r+\frac{\operatorname{Im}\left(k \beta-\tau\left[k \beta_{2}\right]\right)}{\tau_{2}}\right)\right]\right) } \\
& y^{-\frac{r}{2}} q^{\frac{r\left[k \beta_{2}\right]}{2 k}} q^{-\frac{r^{2}}{4 k}} \theta_{r, k}\left(\tau,-\frac{\alpha}{k}+\frac{\beta}{2}-\frac{\tau\left[k \beta_{2}\right]}{2 k}\right) .
\end{aligned}
$$


Consider the argument of the error function: recalling that $\beta=\beta_{1}+\tau \beta_{2}$, we find that

$$
r+\frac{1}{\tau_{2}} \operatorname{Im}\left(k \beta-\tau\left[k \beta_{2}\right]\right)=r+\left(k \beta_{2}-\left[k \beta_{2}\right]\right)
$$

We therefore obtain the part of $k \beta_{2}$ that lies in the interval $(0,1)$, which we will denote by $\gamma_{2}=k \beta_{2}-\left[k \beta_{2}\right]$. We then have:

$$
\begin{array}{r}
\mathcal{R}_{1, k}=-\frac{z^{-1}}{2} \sum_{r \in \mathbb{Z}}\left(\operatorname{sgn}\left(r+\frac{1}{2}\right)-\operatorname{Erf}\left[\sqrt{\frac{\pi \tau_{2}}{k}}\left(r+\gamma_{2}\right)\right]\right) \\
\times y^{-\frac{r}{2}} q^{\frac{\left.r k k \beta_{2}\right]}{2 k}} q^{-\frac{r^{2}}{4 k}} \theta_{r, k}\left(\tau,-\frac{\alpha}{k}+\frac{\beta}{2}-\frac{\left[\tau k \beta_{2}\right]}{2 k}\right)
\end{array}
$$

We now add and subtract $\operatorname{sgn}\left(r+\gamma_{2}\right)$ in the parenthesis and observe that

$$
\sum_{r \in \mathbb{Z}}\left(\operatorname{sgn}\left(r+\frac{1}{2}\right)-\operatorname{sgn}\left(r+\gamma_{2}\right)\right) f(r)=0,
$$

except when $\gamma_{2}=0$ (due to the convention $\operatorname{sgn}(0)=0$ ). Restricting to $\gamma_{2} \neq 0$ - the special case can be treated analogously -, this leads to

$$
\begin{aligned}
\mathcal{R}_{1, k}= & -\frac{z^{-1}}{2} \sum_{r \in \mathbb{Z}}\left(\operatorname{sgn}\left(r+\gamma_{2}\right)-\operatorname{Erf}\left[\sqrt{\frac{\pi \tau_{2}}{k}}\left(r+\gamma_{2}\right)\right]\right) \\
& \times y^{-\frac{r}{2}} q^{\frac{\left.r k \beta_{2}\right]}{2 k}} q^{-\frac{r^{2}}{4 k}} \theta_{r, k}\left(\tau,-\frac{\alpha}{k}+\frac{\beta}{2}-\tau \frac{\left[k \beta_{2}\right]}{2 k}\right) .
\end{aligned}
$$

Using the integral (D.6) we can write the remainder $\mathcal{R}$ as

$$
\mathcal{R}_{1, k}=-\frac{z^{-1}}{\pi} \sum_{r \in \mathbb{Z}} \int_{\mathbb{R}+i \epsilon} \frac{d s}{2 i s+r+\gamma_{2}}(q \bar{q})^{\frac{s^{2}}{k}+\frac{\left(r+\gamma_{2}\right)^{2}}{4 k}} y^{-\frac{r}{2}} q^{\frac{r\left[k \beta_{2}\right]}{2 k}} q^{-\frac{r^{2}}{4 k}} \theta_{r, k}\left(\tau,-\frac{\alpha}{k}+\frac{\beta}{2}-\tau \frac{\left[k \beta_{2}\right]}{2 k}\right) .
$$

We use the definition of the theta function in (D.5), setting $j=w$ and also relabel $r=-v$ to obtain:

$$
\mathcal{R}_{1, k}=-\frac{z^{-1}}{\pi} \sum_{v \in \mathbb{Z}} \sum_{w \in \mathbb{Z}} q^{k w^{2}-v w} z^{-2 w+\frac{v}{k}} y^{k w} q^{-w\left[k \beta_{2}\right]} \int_{\mathbb{R}} \frac{d s}{2 i s-v+\gamma_{2}}(q \bar{q})^{\frac{s^{2}}{k}+\frac{\left(v-\gamma_{2}\right)^{2}}{4 k}} .
$$

Finally, we shift the variable $v$ to $v=\tilde{v}-\left[k \beta_{2}\right]$ and obtain (after dropping the tilde):

$$
\mathcal{R}_{1, k}=\frac{z^{-1-\frac{\left[k \beta_{2}\right]}{k}}}{\pi} \sum_{v \in \mathbb{Z}} \sum_{w \in \mathbb{Z}} q^{k w^{2}-v w} z^{-2 w+\frac{v}{k}} y^{k w} \int_{\mathbb{R}} \frac{d s}{2 i s+v-k \beta_{2}}(q \bar{q})^{\frac{s^{2}}{k}+\frac{\left(v-k \beta_{2}\right)^{2}}{4 k}} .
$$

Here we have also flipped the sign of $s$ in the integral that picks up an extra sign. To summarize, the modularly completed Appell-Lerch sum $\widehat{\mathcal{A}}_{1, k}$ of [9] can be written as the sum of the following holomorphic and remainder terms

$$
\begin{aligned}
& \mathcal{A}_{1, k}=z^{-1} \sum_{w \in \mathbb{Z}} \frac{q^{k w^{2}}\left(z^{-2} y^{k} q^{-\left[k \beta_{2}\right]}\right)^{w}}{1-z^{-\frac{1}{k}} q^{w}} \\
& \mathcal{R}_{1, k}=\frac{z^{-1-\frac{\left[k \beta_{2}\right]}{k}}}{\pi} \sum_{v \in \mathbb{Z}} \sum_{w \in \mathbb{Z}} q^{k w^{2}-v w} z^{-2 w+\frac{v}{k}} y^{k w} \int_{\mathbb{R}} \frac{d s}{2 i s+v-k \beta_{2}}(q \bar{q})^{\frac{s^{2}}{k}+\frac{\left(v-k \beta_{2}\right)^{2}}{4 k}} .
\end{aligned}
$$


If we further multiply this result by the appropriate overall factor we obtain precisely the elliptic genus of the supersymmetric Liouville theory, as in equations (2.12) and (2.13). Moreover, we can use the periodicity properties of the completed Appell-Lerch sum $\widehat{\mathcal{A}}_{1, k}$, written out in (2.18), as well as the even parity property of the elliptic genus, to simplify the final result:

$$
\begin{aligned}
\chi_{L}(\tau, \alpha, \beta) & =\frac{i \theta_{11}(\tau,-\alpha)}{\eta^{3}} z^{1+\frac{\left[k \beta_{2}\right]}{k}} \widehat{\mathcal{A}}_{1, k}\left(\tau,-\frac{\alpha}{k},-2 \alpha+k \beta-k \tau-\tau\left[k \beta_{2}\right]\right) \\
& =\frac{i \theta_{11}(\tau,-\alpha)}{\eta^{3}} \widehat{\mathcal{A}}_{1, k}\left(\tau,-\frac{\alpha}{k},-2 \alpha+k \beta\right) \\
& =\frac{i \theta_{11}(\tau, \alpha)}{\eta^{3}} \widehat{\mathcal{A}}_{1, k}\left(\tau, \frac{\alpha}{k}, 2 \alpha-k \beta\right) .
\end{aligned}
$$

This agrees with [4] and completes the identification of the elliptic genus as the modular completed Appell-Lerch sum for complexified chemical potentials.

We see from the final expression that an alternative appropriate identification of variables is $[2,4]$ :

$$
u=\frac{\alpha}{k}, \quad v=2 \alpha-k \beta .
$$

Yet another road to the same result is obtained if one starts out with the identification of variables

$$
u=\frac{\alpha}{k}, \quad v=2 \alpha-k \tau-k \beta .
$$

Note that the corresponding Appell-Lerch sum differs from the holomorphic part of the elliptic genus in equation (2.12). One then adds the difference to the holomorphic part, and subtracts the difference from the remainder term. The latter term can be written as a sum over the difference of sign functions (see e.g. [8]). These various forms of identifications of variables, use of the periodicity formulas, as well as additions and subtractions code some interesting physics that we explore in the next subsection.

\subsection{Supersymmetric quantum mechanics and wall crossing}

We have obtained a Hamiltonian form for the elliptic genus, and matched it onto results in mathematics. We now wish to interpret these formulae in physical terms. We indicate at least two interesting phenomena. First of all we note that the final formula for the Hamiltonian form of the elliptic genus, equations (2.12) and (2.13), factorizes into a part which we can associate to a free oscillator sum over all generators of the $N=2$ superconformal algebra on the left, multiplied by an Appell-Lerch sum, which is associated to weighted traces in the radial supersymmetric quantum mechanics problems associated to the right-moving primaries $[2,4,23]$.

Consider the remainder term (2.13) first:

$$
\chi_{L, r e m}(\tau, \alpha, \beta)=\frac{i \theta_{11}(\tau,-\alpha)}{\pi \eta^{3}(\tau)} \sum_{n, w} \int_{\mathbb{R}} \frac{d s}{2 i s+n+k w-k \beta_{2}} z^{\frac{n-k w}{k}} y^{k w} q^{-n w}(q \bar{q})^{\frac{s^{2}}{k}+\frac{\left(n+k w-k \beta_{2}\right)^{2}}{4 k}} .
$$

We note a shift in the right-moving momentum due to the imaginary part $\beta_{2}$ of the chemical potential for angular momentum. Indeed, there is an extra term in the Lagrangian due to 
the angular momentum operator insertion which is proportional (as far as the right-movers are concerned) to the right-moving angular momentum. This term shifts the definition of the right-moving momentum, which in turn shifts the definition of the right-moving supercharge. It therefore influences the measure to be the one indicated in equation (2.38). The right-moving Hamiltonian, a function of the right-moving momentum squared, is shifted as well, as can be seen from the exponent of $\bar{q}$ in equation (2.38):

$$
\tilde{L}_{0}-\frac{c}{24}=\frac{s^{2}}{k}+\frac{\left(n+k w-k \beta_{2}\right)^{2}}{4 k} .
$$

Due to the correlated shift in the right-moving supercharge and right-moving Hamiltonian, we see that the pole contributions are still holomorphic, right-moving ground state contributions. The contributions to the left-moving momentum are fixed by the fact that a continuous deformation keeps the locality condition $L_{0}-\tilde{L}_{0}=-n w$ intact. $^{6}$

The second phenomenon, and in fact most phenomena discussed in this paper, is a consequence of the first. We note that the imaginary part $\beta_{2}$ in the angular momentum chemical potential also influences the holomorphic contribution to the elliptic genus:

$\chi_{L, h o l}(\tau, \alpha, \beta)=-\sum_{m, w} \sum_{v=-(k-1)+\left[k \beta_{2}\right]}^{\left[k \beta_{2}\right]}(-1)^{m} q^{(m-1 / 2)^{2} / 2} z^{m-1 / 2} S_{m+k w-1} z^{v / k-2 w} y^{k w} q^{k w^{2}-v w}$.

The $k$ poles in the radial momentum $s$ plane that we pick up are a function of the size of $\beta_{2}$. Whenever $k \beta_{2}$ crosses an integer value, we will see a subtraction and addition to our holomorphic right-moving ground state sum, as can be seen from equation (2.40).

One can also understand this phenomenon from the perspective of the radial rightmoving supersymmetric quantum mechanics (see [22] and appendix C). This is a consequence of the fact that the constant term in the supercharge, associated to the right-moving momentum, flips sign as a function of $\beta_{2}$, rendering a given ground state either normalizable or not. ${ }^{7}$ For integer $k \beta_{2}$, this influences the sum over the right-moving momentum $n+k w$, both in its upper and lower bound. The integer number $k$ of extended $N=2$ superconformal character contributions to the elliptic genus (and in particular the Witten index of the model) remains unchanged under these deformations. As one radial quantum mechanics problem looses a state, another one gains a state. Still, we see jumps in the bound state spectrum, and in particular the R-charges of the Ramond sector ground states whose extended characters contribute to the holomorphic part of the elliptic genus.

As explained below equation (2.12), one can in fact write the holomorphic piece

\footnotetext{
${ }^{6}$ For future purposes, we have recalled some properties of the right-moving supersymmetric quantum mechanics in appendix $\mathrm{C}$ (which in turn is based on e.g. [22]). Our discussion in words can be followed in technical detail by making the identifications $\tilde{L_{0}}-c / 24=H, s^{2} / k=p^{2}, \Phi_{0}^{2}=\left(v-k \beta_{2}\right)^{2} /(4 k)$ between the variables here and those in appendix C. As in [23], each value of the right-moving momentum $v$ gives rise to one radial supersymmetric quantum mechanical system.

${ }^{7}\left(\partial_{x}+\Phi_{0}\right) \psi(x)=0$ for $x \in[0, \infty]$ gives rise to a state state $\psi(x) \propto e^{-\Phi_{0} x}$ which is normalizable or not depending on the sign of the constant $\Phi_{0}$. The conjugate supercharge exhibits a conjugate phenomenon.
} 
schematically as follows:

$$
\chi_{L, h o l}=\sum_{\substack{\text { spectral } \\ \text { flow }}} \sum_{\ell=0}^{k-1}\left[\frac{i \theta_{11}(\tau,-\alpha)}{\eta^{3}(\tau)} z^{\frac{\left[k \beta_{2}\right]-\ell}{k}} \frac{1}{1-z^{-1}}\right]_{\substack{\text { spectral } \\ \text { flow }}} .
$$

The term in the parenthesis is an $\mathcal{N}=2$ superconformal character in the Ramond sector, where the R-charge of the ground state, on which the character is built, is given by $Q_{R}=$ $\frac{1}{2}-\frac{\ell}{k}+\frac{\left[k \beta_{2}\right]}{k}$. The discrete sum is over the Ramond ground states while the final sum is over all states obtained by spectral flow of the ground states by $k w$ units, with $w$ being an integer. This expression shows clearly that the bound state spectrum jumps across the walls where $k \beta_{2}$ is integer.

\section{A model for higher order Appell-Lerch sums}

In this section, we perform a modular covariant differentiation of the elliptic genus to obtain more general Appell-Lerch sums and their modular completions. The analysis carried out in the previous section will then enable us to provide a Hamiltonian interpretation for the modular completions of these higher order Appell-Lerch sums.

\subsection{Modular covariant derivatives}

We confirmed in section 2 that the Liouville elliptic genus $\chi_{L}$ with complexified chemical potentials is proportional to a (completed) Appell-Lerch sum. It thus transforms as a Jacobi form under modular transformations. In this section, we temporarily strip away the $i \theta_{11} / \eta$ prefactor from the elliptic genus (2.35). We can then study modular covariant derivatives of Appell-Lerch sums, following [8]. For the isolated Appell-Lerch sum relevant to us, we have the modular transformation property (see equation (2.18)):

$$
\widehat{\mathcal{A}}_{1, k}\left(\frac{a \tau+b}{c \tau+d}, \frac{u}{c \tau+d}, \frac{v}{c \tau+d}\right)=(c \tau+d) e^{\frac{2 \pi i c}{c \tau+d}\left(v u-k u^{2}\right)} \widehat{\mathcal{A}}_{1, k}(\tau, u, v) .
$$

The chemical potentials in the conformal field theory are related to the variables $u$ and $v$ above by the relations

$$
u=\alpha / k, \quad v=2 \alpha-k \beta .
$$

For our purposes, it is convenient to calculate in terms of the conformal field theory variables $(\alpha, \beta)$. To make this less cumbersome, we introduce the notation:

$$
\widehat{\mathcal{I}}_{1, k}(\tau, \alpha, \beta)=\widehat{\mathcal{A}}_{1, k}\left(\tau, \frac{\alpha}{k}, 2 \alpha-k \beta\right) .
$$

The notation $\widehat{\mathcal{I}}$ is a reminder of the fact that this quantity codes generalized Witten indices of an infinite set of radial supersymmetric quantum mechanics problems [2, 4, 23]. In this notation, we have:

$$
\widehat{\mathcal{I}}_{1, k}\left(\frac{a \tau+b}{c \tau+d}, \frac{\alpha}{c \tau+d}, \frac{\beta}{c \tau+d}\right)=(c \tau+d) e^{\frac{2 \pi i c}{c \tau+d}\left(\frac{\alpha^{2}}{k}-\alpha \beta\right)} \widehat{\mathcal{I}}_{1, k}(\tau, \alpha, \beta) .
$$


This can equally well be derived from the modular transformation of the elliptic genus by stripping away the modular properties of the theta- and eta-functions.

To obtain a new interesting index [8], we act with the derivative operator

$$
\mathcal{D}=\frac{1}{2 \pi i}\left[2 \frac{d}{d \beta}+k \frac{d}{d \alpha}\right]
$$

on both sides of equation (3.4) to obtain

$$
\begin{aligned}
\mathcal{D} \cdot \widehat{\mathcal{I}}_{1, k}\left(\frac{a \tau+b}{c \tau+d}, \frac{\alpha}{c \tau+d}, \frac{\beta}{c \tau+d}\right)=(c \tau+d) e^{\frac{2 \pi i c}{c \tau+d}\left(\frac{\alpha^{2}}{k}-\alpha \beta\right)} \mathcal{D} \cdot \widehat{\mathcal{I}}_{1, k}(\tau, \alpha, \beta) \\
-c k \beta e^{\frac{2 \pi i c}{c \tau+d}\left(\frac{\alpha^{2}}{k}-\alpha \beta\right)} \widehat{\mathcal{I}}_{1, k}(\tau, \alpha, \beta) .
\end{aligned}
$$

In order to compensate for the anomalous second term, consider the modular transformation property of the non-holomorphic expression - recall that $\beta_{2}=\frac{\operatorname{Im}(\beta)}{\tau_{2}}-$ :

$$
\beta_{2} \rightarrow(c \tau+d) \beta_{2}-c \beta .
$$

It follows that the combination

$$
\widehat{\mathcal{I}}_{2, k}(\tau, \alpha, \beta)=\left(\mathcal{D}-k \beta_{2}\right) \cdot \widehat{\mathcal{I}}_{1, k}(\tau, \alpha, \beta)
$$

is a three variable function that transforms modularly: ${ }^{8}$

$$
\widehat{\mathcal{I}}_{2, k}\left(\frac{a \tau+b}{c \tau+d}, \frac{\alpha}{c \tau+d}, \frac{\beta}{c \tau+d}\right)=(c \tau+d)^{2} e^{\frac{2 \pi i c}{c \tau+d} \frac{\alpha^{2}}{k}-\alpha \beta} \widehat{\mathcal{I}}_{2, k}(\tau, \alpha, \beta) .
$$

This technique was used in [8] to obtain higher weight Appell-Lerch sums and their modular completions. The differentiation process will augment the order of the denominator in the Appell-Lerch sum. Since the covariantization does not depend upon the weight of the form on which the derivative acts, one can continue this process and obtain higher weight Jacobi forms iteratively

$$
\widehat{\mathcal{I}}_{n, k}(\tau, \alpha, \beta)=\left(\mathcal{D}-k \beta_{2}\right) \cdot \widehat{\mathcal{I}}_{n-1, k}(\tau, \alpha, \beta),
$$

where the label $n$ specifies the weight of the modular form.

Here, we are interested in providing a Hamiltonian interpretation for the Jacobi forms $\widehat{\mathcal{I}}_{n, k}(\tau, \alpha)$, obtained by setting $\beta=0$, for both their holomorphic and remainder terms. We will also provide a microscopic model for the degrees of freedom coded in these generalized indices.

\subsection{Completed Appell-Lerch sums as state space sums}

In this section we will exhibit the decomposition of the completed Jacobi form $\widehat{\mathcal{I}}_{n, k}$ into a holomorphic piece and a remainder. We use the integral representation naturally provided by the Liouville elliptic genus, stripped of theta- and eta-function factors. Since the integrals allow for a radial supersymmetric quantum mechanics interpretation, this will lead to a natural Hamiltonian interpretation for the Appell-Lerch sums and their modular completions.

\footnotetext{
${ }^{8}$ Other covariant differentiations to higher weight modular forms exist.
} 


\section{The Hamiltonian viewpoint}

Consider the Appell-Lerch sum $\mathcal{I}_{1, k}$ written as in equation (2.12):

$$
\mathcal{I}_{1, k}=-z^{\left[k \beta_{2}\right] / k} \sum_{w \in \mathbb{Z}} \frac{q^{k w^{2}}\left(z^{-2} y^{k} q^{-\left[k \beta_{2}\right]}\right)^{w}}{1-z^{-\frac{1}{k}} q^{w}} .
$$

The remainder term, expressed as an integration over states, is (see equation (2.13)):

$$
\mathcal{R}_{1, k}=-\frac{1}{\pi} \sum_{v \in \mathbb{Z}} \sum_{w \in \mathbb{Z}} q^{k w^{2}-v w} z^{-2 w+\frac{v}{k}} y^{k w} \int_{\mathbb{R}} \frac{d s}{2 i s+v-k \beta_{2}}(q \bar{q})^{\frac{s^{2}}{k}+\frac{\left(v-k \beta_{2}\right)^{2}}{4 k}} .
$$

To simplify the discussion, we choose $\beta_{2}$ to be in the interval $] 0,1 / k[$. This is equivalent to setting $\left[k \beta_{2}\right]=0$ in what follows. This may look dangerous, since we are planning to take a derivative with respect to $\beta$. Note however that the sum of the terms in equations (3.11) and (3.12) behave well as functions of $\beta$, so that we can ignore this subtlety. This property of continuity and differentiability in $\beta$ is also clear from the expression for the Liouville elliptic genus in path integral form.

$$
\mathcal{I}_{2, k}(\tau, \alpha, \beta)=\mathcal{D} \mathcal{I}_{1, k}=\sum_{w} \frac{q^{w(k w+1)} y^{k w} z^{-\frac{1}{k}-2 w}}{\left(1-z^{-\frac{1}{k}} q^{w}\right)^{2}} .
$$

We will refer to expression (3.13) as the double pole Appell-Lerch sum.

Let us now act with the derivative operator on the integral representation of the remainder:

$$
\begin{aligned}
\mathcal{D} \mathcal{R}_{1, k}=- & \frac{1}{\pi} \sum_{v \in \mathbb{Z}} \sum_{w \in \mathbb{Z}} q^{k w^{2}-v w} z^{-2 w+\frac{v}{k}} y^{k w} \\
& \times \int_{\mathbb{R}} d s\left(-\frac{k}{2 \pi \tau_{2}} \frac{1}{\left(2 i s+v-k \beta_{2}\right)^{2}}+\frac{k \beta_{2}}{2 i s+v-k \beta_{2}}\right)(q \bar{q})^{\frac{s^{2}}{k}+\frac{\left(v-k \beta_{2}\right)^{2}}{4 k}} .
\end{aligned}
$$

The first term in the parenthesis can be rewritten using integration by parts to combine it with the second term:

$$
\mathcal{D} \mathcal{R}_{1, k}=-\frac{1}{\pi} \sum_{v \in \mathbb{Z}} \sum_{w \in \mathbb{Z}} q^{k w^{2}-v w} z^{-2 w+\frac{v}{k}} y^{k w} \int_{\mathbb{R}} d s\left(-1+\frac{v}{2 i s+v-k \beta_{2}}\right)(q \bar{q})^{\frac{s^{2}}{k}+\frac{\left(v-k \beta_{2}\right)^{2}}{4 k}} .
$$

The measure in the second term is identical to that of the remainder in the single pole case and the integral over radial momentum $s$ can be done as before by using equation (D.6). We will now make a brief digression to make contact with the results of [8].

\section{Relation to earlier work}

Setting $\beta=0$ in equation (3.15), we obtain the remainder for $\mathcal{I}_{2, k}$ :

$$
\begin{aligned}
\mathcal{R}_{2, k}(\tau, \alpha)= & \frac{1}{2 \pi} \sqrt{\frac{k}{\tau_{2}}} \sum_{v, w} q^{k w^{2}-v w} z^{-2 w+\frac{v}{k}}(q \bar{q})^{\frac{v^{2}}{4 k}} \\
& -\frac{1}{\pi} \sum_{v, w} v q^{k w^{2}-v w} z^{-2 w+\frac{v}{k}} \int_{\mathbb{R}} \frac{d s}{2 i s+v}(q \bar{q})^{\frac{s^{2}}{k}+\frac{v^{2}}{4 k}}
\end{aligned}
$$


Using the definition of the theta function with finite index and the integral (D.6), we find

$$
\begin{aligned}
\mathcal{R}_{2, k}(\tau, \alpha)=\frac{1}{2 \pi} \sqrt{\frac{k}{\tau_{2}}} \sum_{v \in \mathbb{Z}} \bar{q}^{\frac{v^{2}}{4 k}} \theta_{v, k}\left(\tau,-\frac{\alpha}{k}\right) \\
-\frac{1}{2} \sum_{v \in \mathbb{Z}} v q^{-\frac{v^{2}}{4 k}} \theta_{v, k}\left(\tau,-\frac{\alpha}{k}\right)\left(\operatorname{sgn}(v)-\operatorname{Erf}\left[\sqrt{\left.\left.\frac{\pi \tau_{2}}{k} v\right]\right),}\right.\right.
\end{aligned}
$$

which is equal to:

$$
\mathcal{R}_{2, k}(\tau, \alpha)=\frac{1}{2 \pi} \sqrt{\frac{k}{\tau_{2}}} \sum_{v \in \mathbb{Z}} \bar{q}^{\frac{v^{2}}{4 k}} \theta_{v, k}\left(\tau,-\frac{\alpha}{k}\right)-\frac{1}{2} \sum_{v \in \mathbb{Z}}|v| q^{-\frac{v^{2}}{4 k}} \theta_{v, k}\left(\tau,-\frac{\alpha}{k}\right) \operatorname{Erfc}\left[\sqrt{\frac{\pi \tau_{2}}{k}}|v|\right] .
$$

In order to compare this result with the one in [8], we split the variable $v \in \mathbb{Z}$ as

$$
v \equiv 2 k N+\ell, \quad \text { with } \quad N \in \mathbb{Z}, \ell \in \mathbb{Z}_{2 k},
$$

and define

$$
\lambda \equiv \frac{v}{2 k}=N+\frac{\ell}{2 k} .
$$

In terms of these new variables, the expression (3.18) can be rewritten as

$$
\mathcal{R}_{2, k}(\tau, \alpha)=k \sum_{\ell \in \mathbb{Z}_{2 k}} \theta_{l, k}\left(\tau,-\frac{\alpha}{k}\right) \sum_{\lambda \in \mathbb{Z}+\frac{\ell}{2 k}}\left(\frac{1}{2 \pi \sqrt{k \tau_{2}}} \bar{q}^{k \lambda^{2}}-|\lambda| q^{-k \lambda^{2}} \operatorname{Erfc}\left[2|\lambda| \sqrt{\pi \tau_{2} k}\right]\right) .
$$

This matches the remainder in [8] using the map between chemical potentials.

\section{The Hamiltonian interpretation}

We return now to the Hamiltonian interpretation of the covariantly derived Appell-Lerch sums. As recalled from $[2,4,23]$ in subsection 2.6, there is an interpretation of the bound state sum, as well as the remainder integral, in terms of supersymmetric quantum mechanics systems labeled by the right-moving momentum $v=n+k w \cdot{ }^{9}$ It is therefore natural to express the remainder as a sum over the right-moving momentum quantum number:

$$
\mathcal{R}_{1, k}(\tau, \alpha, \beta)=\sum_{v} S_{1, k}(\tau, \alpha, \beta, v)
$$

where we introduced

$$
S_{1, k}(\tau, \alpha, \beta, v)=-\frac{1}{\pi} \sum_{w \in \mathbb{Z}} q^{k w^{2}-v w} z^{-2 w+\frac{v}{k}} y^{k w} \int_{\mathbb{R}} \frac{d s}{2 i s+v-k \beta_{2}}(q \bar{q})^{\frac{s^{2}}{4 k}+\frac{\left(v-k \beta_{2}\right)^{2}}{4 k}} .
$$

${ }^{9}$ This interpretation does not depend on the free oscillator sum $i \theta_{11} / \eta^{3}$ over the modes of the $N=2$ superconformal algebra. It has a more universal character as argued in [16]. This is true even at finite level $k$ as can be seen from the results of [23]. 
From equation (3.15), it is clear that the covariant derivative acts on $S_{1, k}(v)$, which is the contribution from the continuous spectrum, as follows:

$$
\left(\mathcal{D} S_{1, k}\right)(\tau, \alpha, \beta, v)=v S_{1, k}(\tau, \alpha, \beta, v)+Y_{2, k}(\tau, \alpha, \beta, v) .
$$

Here we have introduced the notation $Y_{2, k}$ to denote an ordinary partition sum obtained by integrating over the radial momentum:

$$
Y_{2, k}(\tau, \alpha, \beta, v)=\frac{1}{\pi} \sqrt{\frac{k}{\tau_{2}}} \sum_{w \in \mathbb{Z}} q^{k w^{2}-v w} z^{-2 w+\frac{v}{k}} y^{k w}(q \bar{q})^{\frac{\left(v-k \beta_{2}\right)^{2}}{4 k}} .
$$

We can finally write the holomorphic and remainder pieces of the completed Jacobi form $\widehat{\mathcal{I}}_{2, k}$ in a compact form:

$$
\begin{aligned}
\mathcal{I}_{2, k}(\tau, \alpha, \beta) & =\sum_{w} \frac{q^{w(k w+1)} y^{k w} z^{-\frac{1}{k}-2 w}}{\left(1-z^{-\frac{1}{k}} q^{w}\right)^{2}} \\
\mathcal{R}_{2, k}(\tau, \alpha, \beta) & =-k \beta_{2} \widehat{\mathcal{I}}_{1, k}(\tau, \alpha, \beta)+\sum_{v}\left(v S_{1, k}(\tau, \alpha, \beta, v)+Y_{2, k}(\tau, \alpha, \beta, v)\right) .
\end{aligned}
$$

Our derivation clarifies the Hamiltonian interpretation of these expressions. The AppellLerch sum can be interpreted as an index sum with a right-moving momentum insertion. This is consistent with the wedge sum formula for the Appell-Lerch sum [8]:

$$
\mathcal{I}_{2, k}(\tau, \alpha, 0)=\left(\sum_{w \geq 0} \sum_{v \geq 0}-\sum_{w<0} \sum_{v \leq 0}\right) v q^{k w^{2}+v w} z^{-2 w-\frac{v}{k}} .
$$

The first term in the remainder $\mathcal{R}_{2, k}$ arises from a simple multiplication in the covariant derivative. The second term arises from a right-moving momentum operator insertion. The third term finds its origin in the dependence of the spectral asymmetry as well as the Hamiltonian on the right-moving momentum. This fact is explained in detail in appendix $\mathrm{C}$, equation (C.11). This indeed identifies $Y_{2, k}$ as a partition sum of degrees of freedom living on the asymptotic cylinder. We have thus found a Hamiltonian interpretation of the modular double pole Appell-Lerch sum.

\subsection{Higher order Appell-Lerch sums and their completion}

We can also find a Hamiltonian integral representation for the remainder functions of all higher order Appell-Lerch sums. We act with the covariant derivative $n-1$ times to obtain the holomorphic and remainder piece of $\widehat{\mathcal{I}}_{n, k}$. To compute the explicit expression one needs the action of the covariant derivative on the partition sum $Y_{2, k}$, which is:

$$
\left(\mathcal{D}-k \beta_{2}\right) Y_{2, k}=0 .
$$

This knowledge is sufficient to write down the explicit integral representations of the remainders for any order $n$ and level $k$ in terms of the integral $S_{1, k}$ over the supersymmetric quantum mechanics labeled by the right-moving momentum, and the partition sum $Y_{2, k}$. 
The holomorphic parts of these higher weight Appell-Lerch sums, when $\beta=0$, are written out in [8] in terms of Euler functions. Explicitly, these are given by

$$
\mathcal{I}_{n+1, k}(\tau, \alpha)=\left.\mathcal{D}^{n} \mathcal{I}_{1, k}(\tau, \alpha, \beta)\right|_{\beta=0}=\sum_{w \in \mathbb{Z}} q^{k w^{2}} z^{2 w} \mathcal{E}_{n+1}\left(q^{w} z^{-\frac{1}{k}}\right)
$$

where the $\mathcal{E}_{n}$ are Euler functions, that have a series expansion of the form

$$
\begin{array}{rlrl}
\mathcal{E}_{n+1}(x) & =\sum_{m>0} m^{n} x^{m} \quad \text { if } \quad & |x|>1 \\
& =-\sum_{m<0} m^{n} x^{-m} \quad \text { if } \quad|x|<1 .
\end{array}
$$

The remainders for these generalized Appell-Lerch sums become:

$$
\mathcal{R}_{n+1, k}(\tau, \alpha)=\left.\left(\mathcal{D}-k \beta_{2}\right)^{n} \widehat{\mathcal{I}}_{1, k}(\tau, \alpha, \beta)\right|_{\beta=0}-\mathcal{I}_{n+1, k}(\tau, \alpha)
$$

Explicitly, these are given by

$$
\mathcal{R}_{n+1, k}(\tau, \alpha)=\left.\mathcal{D}^{n} \mathcal{R}_{1, k}\right|_{\beta=0}+\left.\sum_{m} \frac{r_{m}}{m !}\left(\frac{k}{2 \pi \tau_{2}}\right)^{m} \mathcal{D}^{n-2 m} \widehat{\mathcal{I}}_{1, k}\right|_{\beta=0}
$$

The coefficient $r_{m}$ is a product of binomial coefficients, given by

$$
r_{m}=\prod_{\ell=1}^{m}\left(\begin{array}{c}
m-2 \ell \\
2
\end{array}\right)
$$

The first term in equation (3.32) corresponds to insertion of powers of the right-moving momentum, as well as the dependence of the remainder term on the chemical potential through the partition sum $Y_{2, k}$ and its derivatives. The list of other terms arises from the explicit $\beta_{2}$ dependence of the covariant derivative, which needs to be derived and taken into account at every given order, giving rise to the combinatorics exhibited in equation (3.33).

\subsection{Generalized elliptic genera}

In subsection 3.1, we isolated the Appell-Lerch sum $\widehat{I}_{1, k}$ from the elliptic genus $\chi_{L}$ in order to widen the applicability of our Hamiltonian interpretation of the holomorphic and remainder contributions to the higher order Appell-Lerch sums. In this subsection, we return to the context of two-dimensional conformal field theory, in which the radial supersymmetric quantum mechanics models have a direct interpretation in terms of the dynamics of the right-moving superconformal primaries. We thus wish to dress the covariant differentiation of higher order Appell-Lerch sums with the factors corresponding to a free $N=2$ superconformal algebra generator sum for the left-movers.

Recall that the elliptic genus transforms as follows under a modular transformation:

$$
\chi\left(\frac{a \tau+b}{c \tau+d}, \frac{\alpha}{c \tau+d}, \frac{\beta}{c \tau+d}\right)=e^{\left(1+\frac{2}{k}\right) \frac{\pi i \alpha^{2}}{c \tau+d}-\frac{2 \pi i \alpha \beta}{c \tau+d}} \chi(\tau, \alpha, \beta) .
$$


To account for the modular properties of the prefactors, we introduce a modification of the modular covariant derivative (3.5):

$$
\chi^{(1)}(\tau, \alpha, \beta)=\left(\mathcal{D}+\alpha_{2}-k \beta_{2}\right) \chi(\tau, \alpha, \beta) .
$$

We then obtain a weight one Jacobi form that transforms with the same index as the original elliptic genus. The extra factor of $\alpha_{2}$ takes care of the anomalous transformation of the derivative of the theta function. The technical points and Hamiltonian interpretations we proposed in the previous subsections go through for the modularly derived elliptic genus, with one modification. Since we have multiplied in the theta-function which depends on the chemical potential $\alpha$, we find terms that are associated to fermion number operator insertions. This can also be seen by recalling the definition of the elliptic genus in (1.1):

$$
\chi(\tau, \alpha, \beta)=\operatorname{Tr}_{\mathcal{H}}(-1)^{F_{L}+F_{R}} q^{L_{0}-\frac{c}{24}} z^{J_{0}^{R}} y^{P} .
$$

A naive action with the differential operator $\mathcal{D}$ in (3.5), yields the insertion

$$
\mathcal{D} \cdot \chi(\tau, \alpha, \beta) \approx \operatorname{Tr}_{\mathcal{H}}\left[\left(k J_{0}^{R}+2 P\right)(-1)^{F_{L}+F_{R}} q^{L_{0}-\frac{c}{24}} z^{J_{0}^{R}} y^{P}\right] .
$$

Now, the R-charge and the total angular momentum in the coset conformal field theory can be written explicitly in terms of the asymptotic left and right moving momenta as well as the fermion number as follows (see for instance [24]):

$$
J_{0}^{R}=-\frac{2}{k} P_{L}+F_{L} \quad P=P_{L}+P_{R} .
$$

Substituting this into the derivative expression, we find

$$
\mathcal{D} \cdot \chi(\tau, \alpha, \beta) \approx \operatorname{Tr}_{\mathcal{H}}\left[\left(2 P_{R}+k F_{L}\right)(-1)^{F_{L}+F_{R}} q^{L_{0}-\frac{c}{24}} z^{J_{0}^{R}} y^{P}\right] .
$$

We recognize the insertion of the right moving momentum operator we observed in (3.26); in addition we find contributions from the fermion number insertion. The derivation we gave here is naive, since it does not take into account the dependence of the measure on the variables with respect to which we derive. However, since there is no dependence on $\alpha$ in the measure, nor in the right-moving supersymmetric quantum mechanics, the fermion number insertion is the only modification in the picture we painted previously. This is related to the holomorphic dependence of the elliptic genus on the chemical potential for R-charge. We stress that in this conformal field theory context, the origin of the radial supersymmetric quantum mechanics systems for the right-movers can be derived from first principles.

\section{Conclusions}

We have obtained path integral expressions for the Liouville and cigar elliptic genera with complexified chemical potentials, and checked the modular and periodicity properties directly from these expressions. We have also shown that the spectral density asymmetry depends on the imaginary part of the chemical potential $\beta$ for the angular momentum 
on the asymptotic circle. We were able to exhibit a wall-crossing phenomenon in which bound states appear and disappear from the spectrum as a function of the imaginary part of the chemical potential. From the path integral form of the elliptic genus it is clear that the discontinuity in the holomorphic part is mirrored in a discontinuity of the continuum contribution such that the full expression is continuous in $\beta .^{10}$

Furthermore, the generalization to complexified chemical potentials allowed us to take modular covariant derivatives to obtain higher order Appell-Lerch sums and their modular completions. This enabled us to provide a microscopic model for higher order Appell-Lerch sums in which we could give an interpretation to the individual contributions as arising from bound state sums and the continuous spectrum, whose presence results from the spectral asymmetry. Modular derivatives moreover give rise to operator insertions, and a new ingredient in the Hamiltonian interpretation, which is an ordinary partition sum (with trivial spectral weight).

There are many directions for further research. One is the generalization of our analysis to the Jacobi forms of $[20,21,28]$, corresponding to models with more space-time directions (and in the gauged linear sigma-model language, to models with $N$ charged chiral scalar fields). The generalization of the expression (2.2) for higher $N$ is

$$
\begin{aligned}
& \chi_{N}\left(\tau, \alpha, \beta_{i}\right)=\int_{0}^{1} d s_{1} d s_{2} \prod_{i=1}^{N}\left[\frac{\theta_{11}\left(\tau, s_{1} \tau+s_{2}-\alpha-\frac{N \alpha}{k}+\beta_{i}\right)}{\theta_{11}\left(\tau, s_{1} \tau+s_{2}-\frac{N \alpha}{k}+\beta_{i}\right)}\right] \\
& \times \sum_{m, w \in \mathbb{Z}} e^{-2 \pi i s_{2} w} e^{2 \pi i s_{1}(m-N \alpha)} e^{-\frac{\pi}{k \tau_{2}}|m-N \alpha+w \tau|^{2}} .
\end{aligned}
$$

One can check that it is modular covariant and elliptic, with central charge $c=3 N(1+$ $\left.\frac{2 N}{k}\right)$. We can obtain the analogue of the path integral expression (2.5) via double Poisson resummation:

$$
\begin{array}{rl}
\chi_{N}\left(\tau, \alpha, \beta_{i}\right)=k \int_{0}^{1} & d s_{1} d s_{2} \prod_{i=1}^{N}\left[\frac{\theta_{11}\left(\tau, s_{1} \tau+s_{2}-\alpha-\frac{N \alpha}{k}+\beta_{i}\right)}{\theta_{11}\left(\tau, s_{1} \tau+s_{2}-\frac{N \alpha}{k}+\beta_{i}\right)}\right] \\
& \times \sum_{m, w \in \mathbb{Z}} e^{2 \pi i N \alpha w} e^{-2 \pi i N \alpha_{2}\left(\left(s_{1}+w\right) \tau+s_{2}+m\right)} e^{-\frac{\pi k}{\tau_{2}}\left|m+w \tau+\left(s_{1} \tau+s_{2}\right)\right|^{2}} .
\end{array}
$$

This is a good starting point for an analysis of the generalized Hamiltonian interpretation, covariant differentiation, etc. A similar analysis can also be carried out for orbifold and tensor product models.

Another direction for future research was one of the original motivations of this paper. We have given a microscopic interpretation of the higher level Appell-Lerch sums, dressed with further theta- and eta-functions. It should be clear from our analysis that the radial quantum mechanics giving rise to the Appell-Lerch sums exhibits universal features independent of the particular dressing factor. We hope that these features will provide useful hints towards a microscopic interpretation of the mock Jacobi forms arising in the context

\footnotetext{
${ }^{10}$ A similar phenomenon arises in the context of massive $\mathcal{N}=2$ theories in two dimensions [25] and $\mathcal{N}=2$ gauge theories in four dimensions [26].
} 
of microscopic black hole entropy counting, and in particular the modularly completed single or multi-centered black hole partition sums of [8].

\section{Acknowledgments}

We would like to thank Suresh Nampuri, Boris Pioline, Giuseppe Policastro, Ashoke Sen and especially Atish Dabholkar and Sameer Murthy for useful discussions.

\section{A The path integral of the axially gauged coset model}

In this appendix we show how to obtain the path integral expression for the elliptic genus when the chemical potentials $\alpha$ and $\beta$ are taken to be complex. As shown in [4] the path integral can be written in the factorized form

$$
\chi^{P I}(\tau, \alpha)=\int_{\mathbb{C}} \frac{d^{2} u}{2 i \tau_{2}} Z_{g}(\tau, \alpha) Z_{f}(\tau, \alpha) Z_{Y}(\tau, \alpha) Z_{g h}(\tau) Z_{a}(\tau, \alpha) .
$$

We follow the notations and conventions of [4] and refer to that reference for details. The subscripts denote the various sectors: the $\mathrm{SL}(2, \mathbb{R})$ group, the fermions, a compact boson $Y$ and the ghosts. The very last contribution is an anomalous contribution that results from a $\mathrm{U}(1)_{R}$ rotation of the fermions due to their twisted boundary conditions. In the above equation, $u=s_{1} \tau+s_{2}$ is the complexified holonomy that takes values over the entire complex plane.

Here, we will use a slightly different method to obtain the twisted partition functions. ${ }^{11}$ We first write down a modular invariant expression for the path integral in each sector. These are straightforward generalizations of the expressions in [4], with the modification that we make the contribution from each sector modular invariant

$$
\begin{aligned}
Z_{g}(\tau, \alpha)= & \frac{\sqrt{k} \kappa}{\sqrt{\tau_{2}}} \frac{e^{\frac{2 \pi}{\tau_{2}}\left(\operatorname{Im} u-\frac{\operatorname{Im} \alpha}{k}\right)^{2}}}{\left|\theta_{11} \tau, u-\frac{\alpha}{k}\right|^{2}} \\
Z_{f}(\tau, \alpha)= & \frac{1}{\kappa|\eta(\tau)|^{2}}\left[e^{-\frac{\pi}{\tau_{2}}\left(\left|u-\alpha-\frac{\alpha}{k}\right|^{2}-\left(u-\alpha-\frac{\alpha}{k}\right)^{2}\right)} \theta_{11}\left(\tau, u-\alpha-\frac{\alpha}{k}\right)\right] \\
& \times\left[e^{-\frac{\pi}{\tau_{2}}\left(\left|u-\frac{\alpha}{k}\right|^{2}-\left(\bar{u}-\frac{\bar{\alpha}}{k}\right)^{2}\right)} \theta_{11}\left(\bar{\tau}, \bar{u}-\frac{\bar{\alpha}}{k}\right)\right] \\
Z_{Y}(\tau, \alpha)= & \sqrt{\frac{k}{\tau_{2}}} \frac{1}{|\eta(\tau)|^{2}} e^{-\frac{k \pi}{\tau_{2}}|u|^{2}} \\
Z_{g h}= & \tau_{2}|\eta(\tau)|^{4}
\end{aligned}
$$

The further anomaly factor results from a chiral rotation of the fermions that is necessary to write the action as a sum over independent sectors; we find

$$
Z_{a}=e^{\frac{\pi}{2 \tau_{2}}(u \bar{\alpha}-\bar{u} \alpha)} .
$$

\footnotetext{
${ }^{11}$ The method is close to the one used in [27], for instance.
} 
This is, once again, a generalization of the result in [4], now taking the chemical potentials to be complex. The exponent can be understood as coming from a chiral rotation of the fermions that depends on the chemical potential $\alpha$, which measures the R-charge. This leads to an anomaly exponent of the form

$$
\int A^{R} \wedge d Y^{u}
$$

where $A^{R}$ is the background gauge field coupling to the R-charge and $d Y^{u}$ is the differential of the twisted compact boson. Making use of the Riemann bi-linear identity and the definition of the holonomies, we obtain the quoted result. Putting all this together, we find

$$
\chi^{P I}(\tau, \alpha)=e^{\frac{\pi \hat{c}\left(\alpha^{2}-|\alpha|^{2}\right)}{\tau_{2}}} k \int_{\mathbb{C}} \frac{d^{2} u}{2 i \tau_{2}} \frac{\theta_{11}\left(u-\frac{k+1}{k} \alpha\right)}{\theta_{11}\left(u-\frac{\alpha}{k}\right)} e^{-\frac{k \pi}{\tau_{2}}|u|^{2}} e^{-2 \pi i \frac{\alpha_{2}}{\tau_{2}} u} .
$$

One can check that the $\alpha$-dependent prefactor is such that under a modular transformation, the path integral elliptic genus is modular invariant. The Hamiltonian expression for the elliptic genus is simply obtained by omitting this prefactor (see e.g. [27]); we finally find

$$
\chi(\tau, \alpha)=k \int_{\mathbb{C}} \frac{d^{2} u}{2 i \tau_{2}} \frac{\theta_{11}\left(u-\frac{k+1}{k} \alpha\right)}{\theta_{11}\left(u-\frac{\alpha}{k}\right)} e^{-\frac{k \pi}{\tau_{2}}|u|^{2}} e^{-2 \pi i \frac{\alpha_{2}}{\tau_{2}} u} .
$$

This calculation can easily be generalized to include the chemical potential $\beta$.

\section{B Towards the Hamiltonian viewpoint}

In section 2 , we obtained the path integral form of the Liouville elliptic genus $\chi_{L}$ with complexified chemical potentials $\alpha$ and $\beta$

$$
\begin{aligned}
\chi_{L}(\tau, \alpha, \beta)=\sum_{n, m} \int_{0}^{1} d s_{1} d s_{2} \frac{\theta_{11}\left(\tau, s_{1} \tau+s_{2}-\alpha\right)}{\theta_{11}\left(\tau, s_{1} \tau+s_{2}\right)} e^{2 \pi i \alpha \frac{n}{k}} e^{-\frac{k \pi}{\tau_{2}}\left|\left(\frac{n}{k}+s_{1}\right) \tau+\left(\frac{m}{k}+s_{2}\right)+\frac{\alpha}{k}-\beta\right|^{2}} & \times e^{-2 \pi i \alpha_{2}\left(\left(\frac{n}{k}+s_{1}\right) \tau+\left(\frac{m}{k}+s_{2}\right)+\frac{\alpha}{k}-\beta\right)} .
\end{aligned}
$$

In this appendix we will show how to rewrite this in a form that lends itself to a Hamiltonian interpretation, following [2,4] closely. We perform Poisson resummation on the $m$ quantum number in equation (B.1) and find:

$$
\begin{aligned}
\chi_{L}(\tau, \alpha, \beta)=\sqrt{k \tau_{2}} \sum_{n, w} \int_{0}^{1} d s_{1} d s_{2} \frac{\theta_{11}\left(\tau, s_{1} \tau+s_{2}-\alpha\right)}{\theta_{11}\left(\tau, s_{1} \tau+s_{2}\right)} e^{2 \pi i \alpha \frac{n}{k}} e^{-2 \pi i w\left(k s_{2}+\alpha-k \beta_{1}\right)} \\
\times q^{\left(k w-\left(n+k s_{1}-k \beta_{2}\right)\right)^{2} / 4 k} \bar{q}^{\left(k w+\left(n+k s_{1}-k \beta_{2}\right)\right)^{2} / 4 k} .
\end{aligned}
$$

We have shifted the complexified chemical potentials into the exponents to easily expand the denominator theta function following $[2,4]$. Using the expansions in equations (D.1) and (D.3), we obtain

$$
\begin{aligned}
\chi_{L}(\tau, \alpha, \beta)=- & \sqrt{k \tau_{2}} \frac{1}{\eta^{3}} \sum_{m, r, n, w} \int_{0}^{1} d s_{1} d s_{2}(-1)^{m} q^{(m-1 / 2)^{2} / 2}\left(z e^{-2 \pi i s_{2}} q^{-s_{1}}\right)^{m-1 / 2}\left(e^{2 \pi i s_{2}} q^{s_{1}}\right)^{r+1 / 2} \\
& S_{r}(q) z^{n / k} e^{-2 \pi i w\left(k s_{2}+\alpha-k \beta_{1}\right)} q^{\left(k w-\left(n+k s_{1}-k \beta_{2}\right)\right)^{2} / 4 k} \bar{q}^{\left(k w+\left(n+k s_{1}-k \beta_{2}\right)\right)^{2} / 4 k} . \quad \text { (B.3) }
\end{aligned}
$$


The holonomy integral over $s_{2}$ imposes the Gauss constraint $r-m+1=k w$, leading to the simplified expression

$$
\begin{aligned}
\chi_{L}(\tau, \alpha, \beta)=- & \sqrt{k \tau_{2}} \frac{1}{\eta^{3}} \sum_{m, n, w} \int_{0}^{1} d s_{1}(-1)^{m} q^{(m-1 / 2)^{2} / 2} z^{m-1 / 2} S_{m+k w-1}(q) \\
& z^{n / k} e^{-2 \pi i w\left(\alpha-k \beta_{1}\right)} q^{k w s_{1}} q^{\left(k w-\left(n+k s_{1}-k \beta_{2}\right)\right)^{2} / 4 k} \bar{q}^{\left(k w+\left(n+k s_{1}-k \beta_{2}\right)\right)^{2} / 4 k} .
\end{aligned}
$$

Since we know that the power of $\bar{q}$ will be zero for the right-moving ground states, it is advantageous to recombine the exponents of the last two factors as follows:

$$
q^{\left(k w-\left(n+k s_{1}-k \beta_{2}\right)\right)^{2} / 4 k} \bar{q}^{\left(k w+\left(n+k s_{1}-k \beta_{2}\right)\right)^{2} / 4 k}=q^{-n w+k w\left(\beta_{2}-s_{1}\right)}(q \bar{q})^{\left(k w+n+k s_{1}-k \beta_{2}\right)^{2} / 4 k} .
$$

The elliptic genus then simplifies to:

$$
\begin{array}{r}
\chi_{L}(\tau, \alpha, \beta)=-\sqrt{k \tau_{2}} \frac{1}{\eta^{3}} \sum_{m, r, n, w} \int_{0}^{1} d s_{1}(-1)^{m} q^{(m-1 / 2)^{2} / 2} z^{m-1 / 2} S_{m+k w-1}(q) \\
z^{n / k-w} y^{k w} q^{-n w}(q \bar{q})^{\left(k w+n+k s_{1}-k \beta_{2}\right)^{2} / 4 k} .
\end{array}
$$

Next we define the right-moving momentum variable $v \equiv n+k w$ to obtain:

$$
\begin{array}{r}
\chi_{L}(\tau, \alpha, \beta)=-\sqrt{k \tau_{2}} \frac{1}{\eta^{3}} \sum_{m, r, v, w} \int_{0}^{1} d s_{1} \delta_{r-m+1-k w}(-1)^{m} q^{(m-1 / 2)^{2} / 2} z^{m-1 / 2} S_{r}(q) \\
z^{v / k-2 w} y^{k w} q^{k w^{2}-v w}(q \bar{q})^{\left(v+k s_{1}-k \beta_{2}\right)^{2} / 4 k} .
\end{array}
$$

In order to linearize the exponent in $s_{1}$, we use the familiar trick of introducing a new variable $s$, which will later play the role of the momentum along the radial direction:

$$
\begin{array}{r}
\chi_{L}(\tau, \alpha, \beta)=-\frac{2 \tau_{2}}{\eta^{3}} \sum_{m, r, v, w} \int_{0}^{1} d s_{1} \int_{-\infty}^{+\infty} d s(-1)^{m} q^{(m-1 / 2)^{2} / 2} z^{m-1 / 2} S_{m+k w-1}(q) \\
z^{v / k-2 w} y^{k w} q^{k w^{2}-v w}(q \bar{q})^{s_{1}\left(i s+\frac{v}{2}-\frac{k \beta_{2}}{2}\right)+\frac{s^{2}}{k}+\frac{\left(v-k \beta_{2}\right)^{2}}{4 k}} .
\end{array}
$$

This in turn permits us to calculate the integral over the holonomy $s_{1}$ in a convenient form:

$$
\begin{array}{r}
\chi_{L}(\tau, \alpha, \beta)=\frac{1}{\pi \eta^{3}} \sum_{m, v, w} \int_{-\infty}^{+\infty} \frac{d s}{2 i s+v-k \beta_{2}}(-1)^{m} q^{(m-1 / 2)^{2} / 2} z^{m-1 / 2} S_{k w+m-1}(q) \\
z^{v / k-2 w} y^{k w} q^{k w^{2}-v w}\left[(q \bar{q})^{\left(i s+\frac{v}{2}-\frac{k \beta_{2}}{2}\right)}-1\right](q \bar{q})^{\frac{s^{2}}{k}+\frac{\left(v-k \beta_{2}\right)^{2}}{4 k}} .
\end{array}
$$

As in $[2,28]$, there is a simple way to extract the holomorphic piece and remainder term from this expression. We begin with the "1" term in the square parenthesis. In this term, we do a combined shift of variables:

$$
s \longrightarrow s+\frac{i k}{2} \quad v \longrightarrow v+k,
$$


which leads to the following expression:

$$
\begin{array}{r}
\chi_{L}^{1}(\tau, \alpha, \beta)=-\frac{1}{\pi \eta^{3}} \sum_{m, v, w} \int_{\mathbb{R}-\frac{i k}{2}} \frac{d s}{2 i s+v-k \beta_{2}}(-1)^{m} q^{(m-1 / 2)^{2} / 2} z^{m-1 / 2} S_{k w+m-1}(q) z q^{-k w} \\
\left.z^{v / k-2 w} y^{k w} q^{k w^{2}-v w}(q \bar{q})^{\left(i s+\frac{v}{2}-\frac{k \beta_{2}}{2}\right)+\frac{s^{2}}{k}+\frac{\left(v-k \beta_{2}\right)^{2}}{4 k}} . \quad \text { (B. } 11\right)
\end{array}
$$

Now, we use the relation

$$
S_{m+k w-1}(q)=1-S_{-m-k w}(q) .
$$

The part proportional to "1" we again split off and we refer to it as the remainder; we will deal with this piece later. What remains of the term we denote $\chi_{L}^{1, S}$ and it equals

$$
\begin{array}{r}
\chi_{L}^{1, S}(\tau, \alpha, \beta)=\frac{1}{\pi \eta^{3}} \sum_{m, v, w} \int_{\mathbb{R}-\frac{i k}{2}} \frac{d s}{2 i s+v-k \beta_{2}}(-1)^{m} q^{(m-1 / 2)^{2} / 2} z^{m-1 / 2} z q^{-k w} S_{-m-k w}(q) \\
z^{v / k-2 w} y^{k w} q^{k w^{2}-v w}(q \bar{q})^{\left(i s+\frac{v}{2}-\frac{k \beta_{2}}{2}\right)+\frac{s^{2}}{k}+\frac{\left(v-k \beta_{2}\right)^{2}}{4 k}}
\end{array}
$$

We now define $\tilde{m}=m+1$, which allows us to absorb the extra $z$ factor into the $\tilde{m}$ summation. It also leads to an extra overall sign factor. Moreover there is an extra $q$ dependent factor given by $q^{1-\tilde{m}}$; putting all this together we find (after omitting the tilde on $m$ )

$$
\begin{gathered}
\chi_{L}^{1, S}(\tau, \alpha, \beta)=-\frac{1}{\pi \eta^{3}} \sum_{m, v, w} \int_{\mathbb{R}-\frac{i k}{2}} \frac{d s}{2 i s+v-k \beta_{2}}(-1)^{m} q^{(m-1 / 2)^{2} / 2} z^{m-1 / 2} q^{-m+1-k w} S_{-m-k w+1} \\
\left.z^{v / k-2 w} y^{k w} q^{k w^{2}-v w}(q \bar{q})^{\left(i s+\frac{v}{2}-\frac{k \beta_{2}}{2}\right)+\frac{s^{2}}{k}+\frac{\left(v-k \beta_{2}\right)^{2}}{4 k}} . \quad \text { (B. } 14\right)
\end{gathered}
$$

We can now use another relation that the series $S$ satisfies

$$
q^{r} S_{r}(q)=S_{-r}(q)
$$

This allows us to write

$$
\begin{array}{r}
\chi_{L}^{1, S}(\tau, \alpha, \beta)=-\frac{1}{\pi \eta^{3}} \sum_{m, v, w} \int_{\mathbb{R}-\frac{i k}{2}} \frac{d s}{2 i s+v-k \beta_{2}}(-1)^{m} q^{(m-1 / 2)^{2} / 2} z^{m-1 / 2} S_{m+k w-1} \\
z^{v / k-2 w} y^{k w} q^{k w^{2}-v w}(q \bar{q})^{\left(i s+\frac{v}{2}-\frac{k \beta_{2}}{2}\right)+\frac{s^{2}}{k}+\frac{\left(v-k \beta_{2}\right)^{2}}{4 k}} .
\end{array}
$$

What is remarkable is that (B.9) can be combined with $\chi^{1, S}$ above to give a contour integral, which we denote

$$
\begin{array}{r}
\chi_{L, h o l}=\frac{1}{\pi \eta^{3}} \sum_{m, v, w}\left[\int_{\mathbb{R}}-\int_{\mathbb{R}-\frac{i k}{2}}\right] \frac{d s}{2 i s+v-k \beta_{2}}(-1)^{m} q^{(m-1 / 2)^{2} / 2} z^{m-1 / 2} S_{m+k w-1} \\
z^{v / k-2 w} y^{k w} q^{k w^{2}-v w}(q \bar{q})^{\left(i s+\frac{v}{2}-\frac{k \beta_{2}}{2}\right)+\frac{s^{2}}{k}+\frac{\left(v-k \beta_{2}\right)^{2}}{4 k}} .
\end{array}
$$


This is a holomorphic contribution, since, at the location of the poles, the exponent of the non-holomorphic piece vanishes. The piece that is left over will be denoted the remainder and is the " 1 " term in $\chi^{1}$, given by

$$
\begin{array}{r}
\chi_{L, r e m}=-\frac{1}{\pi \eta^{3}} \sum_{m, v, w} \int_{\mathbb{R}-\frac{i k}{2}} \frac{d s}{2 i s+v-k \beta_{2}}(-1)^{m} q^{(m-1 / 2)^{2} / 2} z^{m-1 / 2} z q^{-k w} \\
z^{v / k-2 w} y^{k w} q^{k w^{2}-v w}(q \bar{q})^{\left(i s+\frac{v}{2}-\frac{k \beta_{2}}{2}\right)+\frac{s^{2}}{k}+\frac{\left(v-k \beta_{2}\right)^{2}}{4 k}} .
\end{array}
$$

To obtain the state sum interpretation, we translate the $s$-contour back onto the real axis using the inverse transformations

$$
s \longrightarrow s-\frac{i k}{2} \quad v \longrightarrow v-k .
$$

This also has the effect of removing the imaginary piece from the $(q \bar{q})$ exponent along with the left over $z q^{-k w}$ factor. As a result we obtain (after suitably relabeling the variables):

$$
\chi_{L, r e m}=-\frac{1}{\pi \eta^{3}} \sum_{m, v, w} \int_{\mathbb{R}} \frac{d s}{2 i s+v-k \beta_{2}}(-1)^{m} q^{(m-1 / 2)^{2} / 2} z^{m-1 / 2} z^{v / k-2 w} y^{k w} q^{k w^{2}-v w}(q \bar{q})^{\frac{s^{2}}{k}+\frac{\left(v-k \beta_{2}\right)^{2}}{4 k}} .
$$

Using the theta expansion in (D.1), this can be written as

$$
\chi_{L, r e m}=\frac{i \theta_{11}(\tau,-\alpha)}{\pi \eta^{3}} \sum_{v, w} \int_{\mathbb{R}} \frac{d s}{2 i s+v-k \beta_{2}} z^{v / k-2 w} y^{k w} q^{k w^{2}-v w}(q \bar{q})^{\frac{s^{2}}{k}+\frac{\left(v-k \beta_{2}\right)^{2}}{4 k}} .
$$

The final result for the elliptic genus is therefore a sum of two contributions, in (B.17) and (B.21). We relate these results to the mathematics of completions of Appell-Lerch sums in the bulk of the paper.

\section{Supersymmetric quantum mechanics}

Suppose we have a quantum-mechanical system with Hamiltonian $H$ :

$$
H=p^{2}+\Phi^{2}-\left[\psi^{\dagger}, \psi\right] \Phi^{\prime}(x)
$$

where $\Phi(x)$ is a function of the variable $x$ parameterizing the manifold on which the supersymmetric particle propagates. The commutation relations:

$$
[p, x]=-i \quad\left\{\psi^{\dagger}, \psi\right\}=1
$$

lead to a supersymmetry algebra:

$$
\left\{Q, Q^{\dagger}\right\}=H
$$

for the supercharges

$$
\begin{aligned}
Q & =(p+i \Phi) \psi^{\dagger} \\
Q^{\dagger} & =(p-i \Phi) \psi
\end{aligned}
$$


The Witten index in models with continuous part to their spectrum is given by:

$$
\begin{aligned}
\operatorname{Tr}(-1)^{F} e^{-\beta H}=N_{\text {bos }}(E=0)-N_{\text {ferm }} & (E=0) \\
& +\int_{E_{\text {treshhold }}}^{+\infty} d E e^{-\frac{E}{T}}\left(\rho_{\text {bos }}(E)-\rho_{\text {ferm }}(E)\right) .
\end{aligned}
$$

If we assume the quantum mechanics to live on a half-line (e.g. because of a steep potential at $x=0$ or $x=-\infty$ ), then we have a scattering problem with bosonic and fermionic waves bouncing of the wall. The supercharge relates the wave-functions of these excitations near $x=+\infty$ (where the potential is assumed to take the constant value $\Phi_{0}$ ):

$$
\begin{aligned}
\Psi_{\text {bos }}(x) & \propto e^{i k x}+a_{\text {bos }}(k) e^{-i k x} \\
\Psi_{\text {ferm }}(x) & \propto e^{i k x}+a_{f e r m}(k) e^{-i k x} \\
& \propto\left(i k+\Phi_{0}\right) e^{i k x}+\left(-i k+\Phi_{0}\right) a_{b o s}(k) e^{-i k x} .
\end{aligned}
$$

That leads to a difference in spectral densities equal to:

$$
\begin{aligned}
\rho_{\text {bos }}(k)-\rho_{\text {ferm }}(k) & =\frac{1}{2 \pi i} \frac{d}{d k} \log \frac{a_{\text {bos }}}{a_{\text {ferm }}} \\
& =\frac{1}{2 \pi}\left(\frac{1}{i k+\Phi_{0}}-\frac{1}{i k-\Phi_{0}}\right) .
\end{aligned}
$$

From the behaviour of the potential at infinity, we find that the continuum of states starts at an energy $E=\Phi_{0}$. We can write the (generalized) Witten index as:

$$
\begin{aligned}
\operatorname{Tr}(-1)^{F} e^{-\frac{H}{T}} & =N_{\text {bos }}(E=0)-N_{\text {ferm }}(E=0)+\int_{0}^{+\infty} \frac{d k}{2 \pi}\left(\frac{1}{i k+\Phi_{0}}-\frac{1}{i k-\Phi_{0}}\right) e^{-\frac{E\left(k^{2}\right)}{T}} \\
& =N_{\text {bos }}(E=0)-N_{\text {ferm }}(E=0)+\int_{-\infty}^{+\infty} \frac{d k}{2 \pi} \frac{1}{i k+\Phi_{0}} e^{-\frac{E\left(k^{2}\right)}{T}} .
\end{aligned}
$$

The conserved energy can be evaluated at infinity to be $E=p^{2}+\Phi_{0}^{2}$, such that this simplifies further to:

$$
\operatorname{Tr}(-1)^{F} e^{-\frac{H}{T}}=N_{b o s}(E=0)-N_{\text {ferm }}(E=0)+\int_{-\infty}^{+\infty} \frac{d k}{2 \pi} \frac{1}{i k+\Phi_{0}} e^{-\frac{1}{T}\left(k^{2}+\Phi_{0}^{2}\right)} .
$$

Up to here, the analysis is a minor variation of [22]. What we wish to stress is that, when we derive this weighted trace with respect to the constant $\Phi_{0}$, we find:

$$
\frac{d}{d \Phi_{0}} \operatorname{Tr}(-1)^{F} e^{-\frac{H}{T}}=\int_{-\infty}^{+\infty} \frac{d k}{2 \pi}\left(-\frac{1}{\left(i k+\Phi_{0}\right)^{2}}-\frac{2}{T} \frac{\Phi_{0}}{i k+\Phi_{0}}\right) e^{-\frac{1}{T}\left(k^{2}+\Phi_{0}^{2}\right)},
$$

which through partial integration becomes:

$$
\begin{aligned}
\frac{d}{d \Phi_{0}}\left[\operatorname{Tr}(-1)^{F} e^{-\frac{H}{T}}\right] & =-\frac{1}{\pi T} \int_{-\infty}^{+\infty} d k e^{-\frac{1}{T}\left(k^{2}+\Phi_{0}^{2}\right)} \\
& =-\frac{1}{\sqrt{\pi T}} e^{-\frac{\Phi_{0}^{2}}{T}}
\end{aligned}
$$

Thus, the derivative with respect to the constant in the potential is proportional to an integral over the bosonic continuum, weighted as an ordinary partition function. 


\section{Useful formulae}

The theta function has a power series expansion

$$
\theta_{11}(\tau, \alpha)=-i \sum_{m \in \mathbb{Z}}(-1)^{m} q^{\frac{\left(m-\frac{1}{2}\right)^{2}}{2}} z^{m-\frac{1}{2}} .
$$

The modular and elliptic properties of the theta function are given as follows:

$$
\begin{aligned}
\theta_{11}\left(-\frac{1}{\tau}, \frac{\alpha}{\tau}\right) & =-i(-i \tau)^{\frac{1}{2}} e^{\frac{\pi i \alpha^{2}}{\tau}} \theta_{11}(\tau, \alpha) \\
\theta_{11}(\tau, \alpha+m \tau+n) & =(-1)^{m+n} q^{-\frac{m^{2}}{2}} z^{-m} \theta_{11}(\tau, \alpha) .
\end{aligned}
$$

For the variable $s_{1}$ in the interval $s_{1} \in[0,1]$, the inverse theta function can be expanded as

$$
\begin{aligned}
& \frac{\eta^{3}(\tau)}{i \theta_{11}\left(\tau, s_{1} \tau+s_{2}\right)}=\sum_{r \in \mathbb{Z}}\left(e^{2 \pi i s_{2}} q^{s_{1}}\right)^{r+1 / 2} S_{r}(q), \\
& \text { with } \quad S_{r}(q)=\sum_{n=0}^{\infty}(-1)^{n} q^{\frac{n(n+2 r+1)}{2}}
\end{aligned}
$$

Another useful identity is

$$
\frac{i \theta_{11}(\tau, \alpha)}{1-z q^{p}}=\sum_{m \in \mathbb{Z}}(-1)^{m} q^{\frac{1}{2}\left(m-\frac{1}{2}\right)^{2}} z^{m-\frac{1}{2}} S_{-m+p}(q) .
$$

See e.g. [28] for proofs of these relations. We also use the theta series with finite index, defined as follows:

$$
\sum_{j \in \mathbb{Z}} q^{k\left(j+\frac{r}{2 k}\right)^{2}} z^{2 k\left(j+\frac{r}{2 k}\right)}=\theta_{r, k}(\tau, \alpha)
$$

We have made use of the integral:

$$
\operatorname{sgn}\left(r+\gamma_{2}\right)-\operatorname{Erf}\left(\sqrt{\frac{\pi \tau_{2}}{k}}\left(r+\gamma_{2}\right)\right)=\frac{2}{\pi} \int \frac{d s}{2 i s+r+\gamma_{2}} e^{-\frac{\pi \tau_{2}}{k}\left(4 s^{2}+\left(r+\gamma_{2}\right)^{2}\right)} .
$$

Open Access. This article is distributed under the terms of the Creative Commons Attribution License (CC-BY 4.0), which permits any use, distribution and reproduction in any medium, provided the original author(s) and source are credited.

\section{References}

[1] E. Witten, Elliptic Genera and Quantum Field Theory, Commun. Math. Phys. 109 (1987) 525 [inSPIRE].

[2] J. Troost, The non-compact elliptic genus: mock or modular, JHEP 06 (2010) 104 [arXiv: 1004.3649] [INSPIRE].

[3] T. Eguchi and Y. Sugawara, Non-holomorphic Modular Forms and $\operatorname{SL}(2, \mathbb{R}) / U(1)$ Superconformal Field Theory, JHEP 03 (2011) 107 [arXiv: 1012.5721] [INSPIRE]. 
[4] S.K. Ashok and J. Troost, A Twisted Non-compact Elliptic Genus, JHEP 03 (2011) 067 [arXiv: 1101.1059] [INSPIRE].

[5] A. Hanany, N. Prezas and J. Troost, The Partition function of the two-dimensional black hole conformal field theory, JHEP 04 (2002) 014 [hep-th/0202129] [INSPIRE].

[6] T. Eguchi and Y. Sugawara, $\operatorname{SL}(2, \mathbb{R}) / U(1)$ supercoset and elliptic genera of noncompact Calabi-Yau manifolds, JHEP 05 (2004) 014 [hep-th/0403193] [INSPIRE].

[7] D. Israel, C. Kounnas, A. Pakman and J. Troost, The Partition function of the supersymmetric two-dimensional black hole and little string theory, JHEP 06 (2004) 033 [hep-th/0403237] [INSPIRE].

[8] A. Dabholkar, S. Murthy and D. Zagier, Quantum Black Holes, Wall Crossing and Mock Modular Forms, arXiv:1208.4074 [INSPIRE].

[9] S. Zwegers, Mock Theta functions, PhD thesis, Utrecht University (2002).

[10] D. Zagier, Ramanujan's mock theta functions and their applications d'après Zwegers and Bringmann-Ono, Séminaire Bourbaki (2007) 986.

[11] T. Eguchi and A. Taormina, Character Formulas for the $N=4$ Superconformal Algebra, Phys. Lett. B 200 (1988) 315 [INSPIRE].

[12] A.M. Semikhatov, A. Taormina and I.Y. Tipunin, Higher level Appell functions, modular transformations and characters, math/0311314 [INSPIRE].

[13] T. Eguchi and K. Hikami, Superconformal Algebras and Mock Theta Functions, J. Phys. A 42 (2009) 304010 [arXiv:0812.1151] [inSPIRE].

[14] J. Manschot, Stability and duality in $N=2$ supergravity, Commun. Math. Phys. 299 (2010) 651 [arXiv:0906.1767] [INSPIRE].

[15] A. Giveon, N. Itzhaki and J. Troost, The Black Hole Interior and a Curious Sum Rule, JHEP 03 (2014) 063 [arXiv:1311.5189] [INSPIRE].

[16] A. Giveon, N. Itzhaki and J. Troost, Lessons on Black Holes from the Elliptic Genus, arXiv: 1401.3104 [INSPIRE].

[17] J.A. Harvey and S. Murthy, Moonshine in Fivebrane Spacetimes, JHEP 01 (2014) 146 [arXiv: 1307.7717] [INSPIRE].

[18] G. Lopes Cardoso, M. Cirafici, R. Jorge and S. Nampuri, Indefinite theta functions and black hole partition functions, JHEP 02 (2014) 019 [arXiv: 1309.4428] [INSPIRE].

[19] T. Kawai, Y. Yamada and S.-K. Yang, Elliptic genera and $N=2$ superconformal field theory, Nucl. Phys. B 414 (1994) 191 [hep-th/9306096] [INSPIRE].

[20] S. Murthy, A holomorphic anomaly in the elliptic genus, JHEP 06 (2014) 165 [arXiv: 1311.0918] [INSPIRE].

[21] S.K. Ashok, N. Doroud and J. Troost, Localization and real Jacobi forms, JHEP 04 (2014) 119 [arXiv:1311.1110] [INSPIRE].

[22] R. Akhoury and A. Comtet, Anomalous Behavior of the Witten Index: Exactly Soluble Models, Nucl. Phys. B 246 (1984) 253 [inSPIRE].

[23] S.K. Ashok, S. Nampuri and J. Troost, Counting Strings, Wound and Bound, JHEP 04 (2013) 096 [arXiv:1302.1045] [INSPIRE]. 
[24] D. Israel, A. Pakman and J. Troost, D-branes in $N=2$ Liouville theory and its mirror, Nucl. Phys. B 710 (2005) 529 [hep-th/0405259] [InSPIRE].

[25] S. Cecotti, P. Fendley, K.A. Intriligator and C. Vafa, A New supersymmetric index, Nucl. Phys. B 386 (1992) 405 [hep-th/9204102] [INSPIRE].

[26] B. Pioline, On the Witten index of N=2 four dimensional gauge theories, talk at Mock-modular forms and physics, International Workshop at IMSc, Chennai, April 14-18 2014, based on work by S. Alexandrov, G. W. Moore, A. Neitzke and B. Pioline.

[27] P. Kraus and F. Larsen, Partition functions and elliptic genera from supergravity, JHEP 01 (2007) 002 [hep-th/0607138] [INSPIRE].

[28] S.K. Ashok and J. Troost, Elliptic genera and real Jacobi forms, JHEP 01 (2014) 082 [arXiv: 1310.2124] [INSPIRE]. 